\title{
The effective action of double field theory
}

\author{
Gerardo Aldazabal, ${ }^{a}$ Walter Baron, ${ }^{b}$ Diego Marqués ${ }^{c}$ and Carmen Núñez ${ }^{b, d}$ \\ ${ }^{a}$ Centro Atómico Bariloche, and Instituto Balseiro (CNEA-UNC) and CONICET, \\ 8400 S.C. de Bariloche, Argentina \\ ${ }^{b}$ Instituto de Astronomía y Física del Espacio (CONICET-UBA), \\ C.C. 67 - Suc. 28, 1428 Buenos Aires, Argentina \\ ${ }^{c}$ Institut de Physique Théorique, CEA/ Saclay, \\ 91191 Gif-sur-Yvette Cedex, France \\ ${ }^{d}$ Departamento de Física, FCEN, Universidad de Buenos Aires \\ Buenos Aires, Argentina \\ E-mail: aldazaba@cab.cnea.gov.ar, w_baron@iafe.uba.ar, \\ diego.marques@cea.fr, carmen@iafe.uba.ar
}

ABSTRACT: We perform a generalized Scherk-Schwarz dimensional reduction of Double Field Theory on a twisted double torus. The four dimensional effective action is shown to exactly reproduce the bosonic electric sector of gauged $\mathcal{N}=4$ supergravity. We present explicit expressions for the gaugings in terms of the twists, and analyze the associated string backgrounds. This framework provides a higher dimensional origin of the gaugings in terms of generalized fluxes.

Keywords: Flux compactifications, String Duality

ArXiv EPRINT: 1109.0290 


\section{Contents}

1 Introduction 1

2 Review of double field theory $\quad 3$

3 Scherk-Schwarz dimensional reduction 5

4 The effective action of DFT and gauged $\mathcal{N}=4$ supergravity 10

$\begin{array}{ll}4.1 \text { Review of gauged } \mathcal{N}=4 \text { supergravity } & 11\end{array}$

$\begin{array}{ll}4.2 & \text { Comparison with the effective action of DFT } \\ \end{array}$

$\begin{array}{lll}4.2 .1 & \text { The } O(6,6) \text { sector } & 13\end{array}$

$\begin{array}{lll}4.2 .2 & \text { Including vectors } & 14\end{array}$

$\begin{array}{lll}4.2 .3 & \text { Rescaling symmetry gaugings } & 16\end{array}$

5 (Non)geometric string backgrounds $\quad 17$

$\begin{array}{lll}5.1 & \text { Reduction on a twisted double torus } & 17\end{array}$

$\begin{array}{ll}5.2 \text { Generalized fluxes and superstring compactifications } & 19\end{array}$

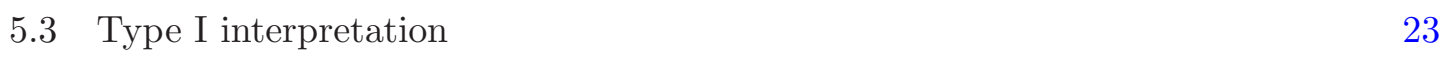

6 Conclusions $\quad 24$

$\begin{array}{ll}\text { A Gauging away } \beta \text {-transformations } & 25\end{array}$

$\begin{array}{lr}\text { B A single flux example } & \mathbf{2 7}\end{array}$

$\begin{array}{lll}\text { B.1 } H \text {-flux } & 27\end{array}$

B.2 $\omega$-flux 28

$\begin{array}{lll}\text { B.3 } Q \text {-flux } & 29\end{array}$

B.4 R-flux 30

\section{Introduction}

Flux compactifications of string theory [1-3] lead to gauged supergravities [4], providing an efficient mechanism of moduli stabilization and spontaneous supersymmetry breaking. An intriguing puzzle is that gauged supergravity contains more gaugings than those that can be reached through geometric compactifications of the different string effective actions or string supergravities. The presence of additional (non-geometric) gaugings suggests that some features of string compactifications have not yet been properly taken into account.

The missing gaugings could be obtained by invoking U-duality arguments at the level of the dimensionally reduced effective actions [5]-[8]. This approach corresponds to the dotted (blue) path in figure 1. Although efficient in generating the full set of gaugings, 


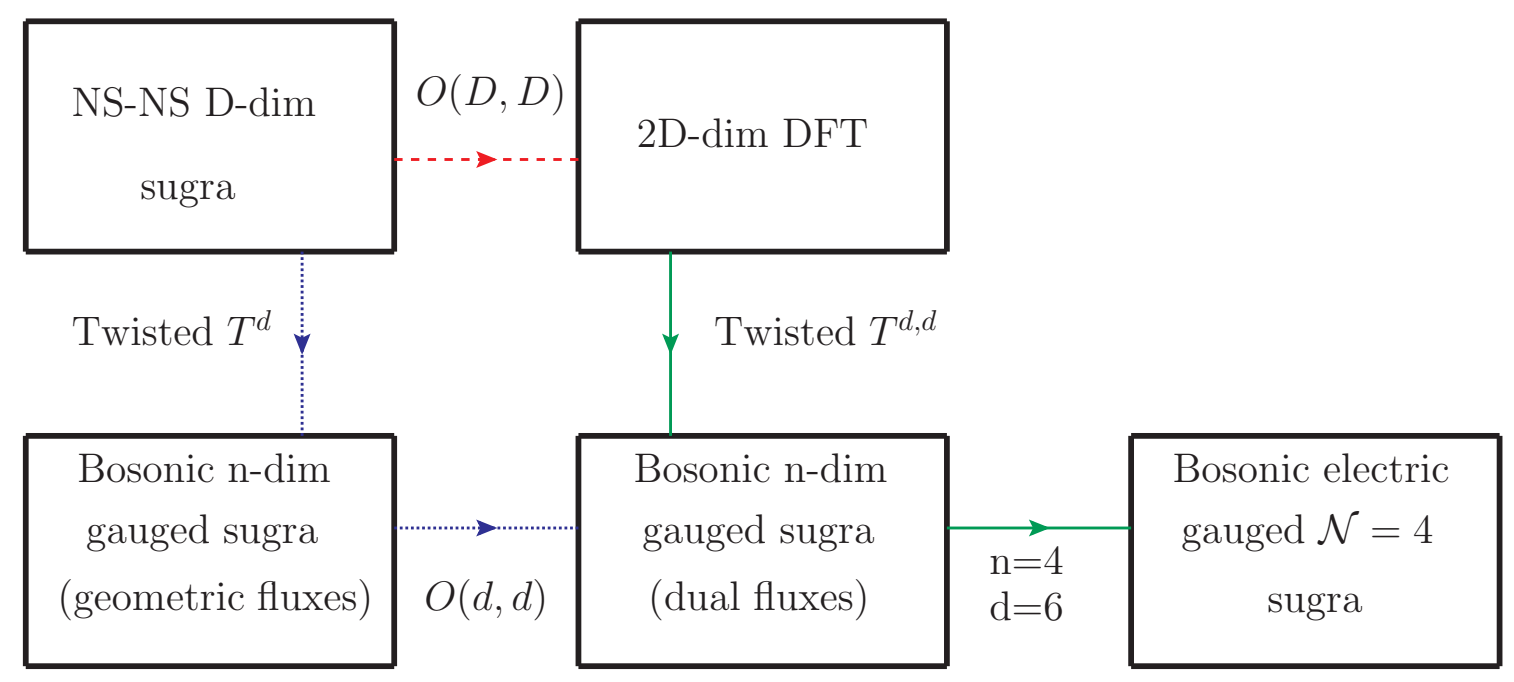

Figure 1. From string supergravity to four dimensional $\mathcal{N}=4$ gauged supergravity.

this procedure presents the disadvantage of leaving many of them unexplained in terms of a higher dimensional origin. For instance, enforcing T-duality invariance requires the introduction of non-geometric fluxes. This can be seen by comparing the effective four dimensional superpotentials arising in orientifold compactifications of type IIA and IIB ten dimensional supergravity actions, where a broader class of internal spaces is needed to have a geometric interpretation.

The concepts of Generalized Complex Geometry [9]-[11], non-geometry [12, 13] and double geometry [14]-[20] were proposed as suitable frameworks to deal with this situation (see also $[21,22]$ for extensions to M-theory). By treating the symmetries of the NSNS antisymmetric tensor field and diffeomorphisms on an equal footing, some of the properties of T-duality are naturally incorporated. Inspired by this approach, a way to obtain the usual contribution of the locally geometric $Q$-flux [23] to the four-dimensional effective action, from a ten dimensional one, was recently proposed in [24].

On the other hand, there have also been attempts to promote U-duality to a symmetry at the level of the higher dimensional supergravity. For T-duality this was successfully achieved by Double Field Theory (DFT), originally formulated in [25]-[28] and extended in [29]-[39]. This attempt is represented in the figure with the dashed (red) arrow. The idea, which we review in section 2 , was to introduce additional coordinates to the standard space-time embedding of closed strings in toroidal backgrounds, dual to winding. In DFT the fields depend on both sets of coordinates and this gives rise to a $2 D$-dimensional theory formulated on a double space. The stringy nature of the theory is manifested in the fact that DFT is T-duality invariant, so it promotes a string duality to a symmetry.

In this work we follow the path indicated by the two solid (green) arrows in figure 1. First, by performing a generalized Scherk-Schwarz dimensional reduction [40] on a twisted double torus we obtain the effective action of DFT. This procedure allows to identify the new degrees of freedom present in DFT as the origin of the missing (non-geometric) gaug- 
ings. The computation, for an arbitrary number of dimensions, is performed in section 3 . The reduced theory is a generalization of that found in [41]. Interestingly enough, it includes geometric fluxes as well as the locally (but not globally) geometric fluxes $Q$ and the locally non-geometric fluxes $R$. Besides, it inherits a remnant gauge symmetry from DFT based on the C-bracket, and a (would be) global $O(d, d)$ invariance which is broken by the fluxes.

In section 4 we show that the four dimensional effective action with six internal dimensions is dual to the bosonic electric sector of gauged $\mathcal{N}=4$ supergravity, as formulated in [42]. This corresponds to the second solid (green) arrow. In order to recover the full set of gaugings, we show how to generalize the Scherk-Schwarz reduction to include the fundamental fluxes $\xi_{+M}$. Additionally, we enhance the $O(D, D)$ symmetry of DFT to $O(D, D+N)$ by adding $N$ generalized vector fields along the lines of [43] and [30].

Section 5 is devoted to the analysis of the string (non)geometric backgrounds and the fluxes they give rise to. We show that the internal space can be thought of as a twisted double torus. Alternative interpretations of our results from the point of view of superstring compactifications are discussed. In particular we show that the resulting effective theory can be seen either as a bosonic sector of heterotic string compactification, extended by T-duality [5, 8], or as a bosonic sector of a Type IIB orientifold compactification [7, 44].

Finally, the conclusions in section 6 contain a summary of our results. We include an appendix illustrating how single-flux string backgrounds are encoded in the twisted double torus.

\section{Review of double field theory}

$D$-dimensional closed strings on toroidal backgrounds carry both momentum and winding, the former being dual to space-time coordinates. Double Field Theory was constructed out of the idea of assigning dual coordinates also to winding. While in string supergravities in $D$ dimensions fields depend only on space-time coordinates, DFT incorporates a dependence also on the coordinates dual to winding. For this reason, it is a theory defined in a doubled space with coordinates $X^{\hat{M}}=\left(X^{\hat{\mu}}, \tilde{X}_{\hat{\mu}}\right)$. The most remarkable feature of this theory is that it is invariant under T-dualities, and more generally under the full $O(D, D)$ group associated to the isometries of the doubled torus. In this way, DFT is a field theory that takes "stringy" features into account by promoting a string duality to a symmetry.

In this section we present a brief review of the generalized metric formulation of DFT, mainly to exhibit the results that we will use throughout the paper. The notation basically coincides with the standard conventions, but we put a hat on all fields and indices to facilitate the reading of the forthcoming sections.

The building block of the theory is the generalized $2 D \times 2 D$ metric

$$
\hat{\mathcal{H}}_{\hat{M} \hat{N}}=\left(\begin{array}{cc}
\hat{g}^{\hat{\mu} \hat{\nu}} & -\hat{g}^{\hat{\mu} \hat{\rho}} \hat{B}_{\hat{\rho} \hat{\nu}} \\
\hat{B}_{\hat{\mu} \hat{\rho}} \hat{g}^{\hat{\rho} \hat{\nu}} & \hat{g}_{\hat{\mu} \hat{\nu}}-\hat{B}_{\hat{\mu} \hat{\rho}} \hat{\rho^{\hat{\rho}} \hat{\sigma}} \hat{B}_{\hat{\sigma} \hat{\nu}}
\end{array}\right)
$$


constructed out of the $D$-dimensional metric $\hat{g}_{\hat{\mu} \hat{\nu}}\left(X^{\hat{\mu}}, \tilde{X}_{\hat{\mu}}\right)$ and the $D$-dimensional 2-form field $\hat{B}_{\hat{\mu} \hat{\nu}}\left(X^{\hat{\mu}}, \tilde{X}_{\hat{\mu}}\right)$. This generalized metric is an element of $O(D, D)$, so it preserves the metric of the group that we take of the following form

$$
\hat{\mathcal{I}}=\left(\begin{array}{cc}
0 & 1_{D} \\
1_{D} & 0
\end{array}\right) .
$$

In addition, the model also contains an $O(D, D)$ invariant dilaton

$$
e^{-2 \hat{d}}=\sqrt{|\hat{g}|} e^{-2 \hat{\phi}} .
$$

The background independent formulation of DFT is defined by an action that can be written in a compact form (up to total derivatives) in terms of a generalized Ricci scalar as

$$
S_{\mathrm{DFT}}=\int d^{D} X d^{D} \tilde{X} e^{-2 \hat{d}} \mathcal{R}(\hat{\mathcal{H}}, \hat{d})
$$

where $\mathcal{R}$ is defined by

$$
\begin{aligned}
\mathcal{R}(\hat{\mathcal{H}}, \hat{d})= & 4 \hat{\mathcal{H}}^{\hat{M} \hat{N}} \partial_{\hat{M}} \partial_{\hat{N}} \hat{d}-\partial_{\hat{M}} \partial_{\hat{N}} \hat{\mathcal{H}}^{\hat{M} \hat{N}}-4 \hat{\mathcal{H}}^{\hat{M} \hat{N}} \partial_{\hat{M}} \hat{d} \partial_{\hat{N}} \hat{d}+4 \partial_{\hat{M}} \hat{\mathcal{H}}^{\hat{M} \hat{N}} \partial_{\hat{N}} \hat{d} \\
& +\frac{1}{8} \hat{\mathcal{H}}^{\hat{M} \hat{N}} \partial_{\hat{M}} \hat{\mathcal{H}}^{\hat{K} \hat{L}} \partial_{\hat{N}} \hat{\mathcal{H}}_{\hat{K} \hat{L}}-\frac{1}{2} \hat{\mathcal{H}}^{\hat{M} \hat{N}} \partial_{\hat{M}} \hat{\mathcal{H}}^{\hat{K} \hat{L}} \partial_{\hat{K}} \hat{\mathcal{H}}_{\hat{N} \hat{L}} .
\end{aligned}
$$

When the coordinates dual to winding are frozen, i.e. $\partial_{\tilde{X}} \bullet=0$, this action reduces to the standard NS-NS bosonic sector of the $D$-dimensional string supergravities. The theory is constrained by a world-sheet level-matching condition that can be written as

$$
\partial_{\hat{M}} \partial^{\hat{M}} \bullet=0,
$$

and the construction of the action (2.4) requires the so called "strong constraint" stating that all fields and their products (represented by - above) must be annihilated by the differential operator (2.6). The strong constraint implies that locally there is always an $O(D, D)$ transformation that rotates into a frame in which fields depend only on half of the coordinates.

Regarding symmetries, the action is manifestly invariant under global $O(D, D)$ transformations acting as

$$
\hat{\mathcal{H}}_{\hat{M} \hat{N}} \rightarrow \hat{U}_{\hat{M}}^{\hat{A}} \hat{\mathcal{H}}_{\hat{A} \hat{B}} \hat{U}_{\hat{N}}^{\hat{B}}, \quad \hat{d} \rightarrow \hat{d}, \quad \hat{U} \in O(D, D) .
$$

In addition it has the following gauge symmetry

$$
\begin{aligned}
\delta_{\hat{\xi}} \hat{d} & =\hat{\xi}^{\hat{M}} \partial_{\hat{M}} \hat{d}-\frac{1}{2} \partial_{\hat{M}} \hat{\xi}^{\hat{M}}, \\
\delta_{\hat{\xi}} \hat{\mathcal{H}}^{\hat{M} \hat{N}} & =\hat{\xi}^{P} \partial_{P} \hat{\mathcal{H}}^{\hat{M} \hat{N}}+\left(\partial^{\hat{M}} \hat{\xi}_{\hat{P}}-\partial_{\hat{P}} \hat{\xi}^{\hat{M}}\right) \hat{\mathcal{H}}^{\hat{P} \hat{N}}+\left(\partial^{\hat{N}} \hat{\xi}_{\hat{P}}-\partial_{\hat{P}} \hat{\xi}^{\hat{N}}\right) \hat{\mathcal{H}}^{\hat{M} \hat{P}} .
\end{aligned}
$$

This transformation rule is an $O(D, D)$ covariant extension of the standard Lie derivative that governs infinitesimal diffeomorphisms. Generalized Lie derivatives acting on vectors can be constructed as

$$
\hat{\mathcal{L}}_{\hat{\xi}} A_{\hat{M}}=\hat{\xi}^{\hat{N}} \partial_{\hat{N}} A_{\hat{M}}+\left(\partial_{\hat{M}} \hat{\xi}^{\hat{N}}-\partial^{\hat{N}} \hat{\xi}_{\hat{M}}\right) A_{\hat{N}},
$$


such that the transformation rules (2.8) read $\delta_{\hat{\xi}} \hat{\mathcal{H}}_{\hat{M} \hat{N}}=\hat{\mathcal{L}}_{\hat{\xi}} \hat{\mathcal{H}}_{\hat{M} \hat{N}}$. The gauge transformations then close under the so-called C-bracket, defined as

$$
\left[\hat{\mathcal{L}}_{\hat{\xi}_{1}}, \hat{\mathcal{L}}_{\hat{\xi}_{2}}\right] A_{\hat{M}}=-\hat{\mathcal{L}}_{\left[\hat{\xi}_{1}, \hat{\xi}_{2}\right]_{\mathrm{C}}} A_{\hat{M}}
$$

where

$$
\left[\hat{\xi}_{1}, \hat{\xi}_{2}\right]_{\mathrm{C}}^{\hat{M}}=2 \hat{\xi_{[1}^{\hat{N}}} \partial_{\hat{N}} \hat{\xi}_{2]}^{\hat{M}}-\hat{\xi}_{[1}^{\hat{P}} \partial^{\hat{M}} \hat{\xi}_{2] \hat{P}}
$$

For further insight on DFT we refer to the original works [25]-[28] and to their many extensions [29]-[39].

\section{Scherk-Schwarz dimensional reduction}

In this section we perform the twisted dimensional reduction of DFT and compute the effective action. The starting point for the compactification procedure is the generalized $2 D$-dimensional double space on which DFT is defined. We identify $n$ of its coordinates with space-time coordinates and other $n$ with their duals. The first step is to compactify this double space-time on a double torus of vanishing dual radius, so that the usual space-time is naturally decompactified. We are therefore effectively left with a $(n+2 d)$-dimensional space $(D=n+d)$ that we compactify on a twisted $T^{d, d}$ torus to obtain the $n$-dimensional effective theory of DFT.

Throughout the paper we use many different indices. The original formulation of DFT in $2 D$-dimensions is based on the usual $D$ "string" coordinates and the remaining $D$ correspond to the dual space. After dimensionally reducing in a $2 d$-dimensional space, the effective action is an $n=D-d$ dimensional theory (provided the coordinates dual to space-time are taken to vanish). Of course, at the end of the day our main interest is in $D=10, d=6$ and $n=3+1$, but since most computations can be performed without explicitly specifying the dimensions, we keep the results as general as possible. We use the following notation:

Curved and tangent indices are respectively

$$
\hat{M}, \hat{N}, \hat{O}, \hat{P}, \cdots \in\{0,1,2, \ldots, 2 D-1\}, \quad \hat{A}, \hat{B}, \hat{C}, \hat{D}, \cdots \in\{0,1,2, \ldots, 2 D-1\},
$$

for the full $2 D$ space;

$$
\hat{\mu}, \hat{\nu}, \hat{\xi}, \hat{\rho}, \hat{\sigma}, \cdots \in\{0,1,2, \ldots, D-1\}, \quad \hat{m}, \hat{n}, \hat{o}, \hat{p}, \hat{q}, \cdots \in\{0,1,2, \ldots, D-1\},
$$

for the $D$-dimensional "stringy" coordinates;

$$
M, N, O, P, \cdots \in\{1,2, \ldots, 2 d\}, \quad A, B, C, D, \cdots \in\{1,2, \ldots, 2 d\},
$$

for $2 d$-dimensional internal and dual coordinates (which we denote by $\mathbb{Y}^{M}$ );

$$
\mu, \nu, \rho, \sigma, \cdots \in\{0,1, \ldots, n-1\}, \quad m, n, p, q, \cdots \in\{0,1, \ldots, n-1\},
$$

for spacetime and

$$
\alpha, \beta, \gamma, \delta, \cdots \in\{1,2, \ldots, d\}, \quad a, b, c, d, \cdots \in\{1,2, \ldots, d\},
$$


for the $d$-dimensional internal indices (with the corresponding coordinates denoted as $y^{\alpha}$ ). Dual coordinates are $\tilde{y}_{\alpha}$ and we usually write $\mathbb{Y}^{A}=\left(\tilde{y}_{a}, y^{a}\right)$.

As we have seen, the degrees of freedom in DFT are represented by an invariant dilaton $\hat{d}$ defined in (2.3) and a generalized $2 D \times 2 D$ metric (2.1), namely

$$
\hat{\mathcal{H}}_{\hat{M} \hat{N}}=\left(\begin{array}{cc}
\hat{\mathcal{H}}^{\hat{\mu} \hat{\nu}} & \hat{\mathcal{H}}^{\hat{\mu}} \\
\hat{\mathcal{H}}_{\hat{\mu}} \hat{\nu} & \hat{\mathcal{H}}_{\hat{\mu} \hat{\nu}}
\end{array}\right)=\left(\begin{array}{cc}
\hat{g}^{\hat{\mu} \hat{\nu}} & -\hat{g}^{\hat{\mu} \hat{\rho}} \hat{B}_{\hat{\rho} \hat{\nu}} \\
\hat{B}_{\hat{\mu} \hat{\rho}} \hat{g}^{\hat{\rho} \hat{\nu}} & \hat{g}_{\hat{\mu} \hat{\nu}}-\hat{B}_{\hat{\mu} \hat{\rho}} \hat{g}^{\hat{\rho} \hat{\sigma}} \hat{B}_{\hat{\sigma} \hat{\nu}}
\end{array}\right) .
$$

This metric can be constructed out of a vielbein with triangular ${ }^{1}$ form, i.e.

$$
\hat{\mathcal{E}}=\left(\begin{array}{cc}
\left(\hat{e}^{-1}\right)^{T} & -\left(\hat{e}^{-1}\right)^{T} \hat{B} \\
0 & \hat{e}
\end{array}\right)
$$

which is related to (3.6) through

$$
\hat{\mathcal{H}}=\hat{\mathcal{E}}^{T} \hat{\eta} \hat{\mathcal{E}}, \quad \hat{\eta}=\left(\begin{array}{cc}
\eta^{-1} & 0 \\
0 & \eta
\end{array}\right)
$$

where $\eta=\operatorname{diag}(-+\cdots+)$.

We have defined the $D$-dimensional fields that parameterize the generalized metric, namely the Kalb-Ramond field $\hat{B}$ and the $D$-bein $\hat{e}$ associated to the metric

$$
\hat{g}_{\hat{\mu} \hat{\nu}}=\hat{e}^{\hat{m}} \hat{\mu} \hat{\eta}_{\hat{m} \hat{n}} \hat{e}^{\hat{n}} \hat{\nu}=\left(\hat{e}^{T} \hat{\eta} \hat{e}\right)_{\hat{\mu} \hat{\nu}} .
$$

These can then be written as usual in terms of the compactified degrees of freedom as

$$
\hat{g}_{\hat{\mu} \hat{\nu}}=\left(\begin{array}{cc}
g_{\mu \nu}+h_{\gamma \delta} \mathcal{A}^{\gamma}{ }_{\mu} \mathcal{A}^{\delta}{ }_{\nu} & \mathcal{A}^{\gamma}{ }_{\mu} h_{\gamma \beta} \\
h_{\alpha \gamma} \mathcal{A}^{\gamma}{ }_{\nu} & h_{\alpha \beta}
\end{array}\right), \quad \hat{B}_{\hat{\mu} \hat{\nu}}=\left(\begin{array}{cc}
\breve{B}_{\mu \nu} & -\breve{\mathcal{B}}_{\beta \mu} \\
\breve{\mathcal{B}}_{\alpha \nu} & b_{\alpha \beta}
\end{array}\right) .
$$

From the $n$-dimensional point of view, the $D$-dimensional metric decomposes into an $n$-dimensional metric $g_{\mu \nu}, d$ vectors $\mathcal{A}_{\mu}^{\alpha}$ and $d(d+1) / 2$ real scalars $h_{\alpha \beta}$. The $B$-field also decomposes into an $n$-dimensional 2-form $B_{\mu \nu}, d$ vectors $\mathcal{B}_{\alpha \mu}$ (which we will further relate to $\breve{B}_{\mu \nu}$ and $\breve{\mathcal{B}}_{\alpha \nu}$ in equation $\left.(3.18)\right)$ and $d(d-1) / 2$ real scalars $b_{\alpha \beta}$.

Having set the structure of the (compactified) fields, we proceed with the ScherkSchwarz twist. The global symmetry of the action that we intend to twist is the $O(D, D)$ invariance defined in (2.7). We perform a $\hat{U} \in O(D, D)$ transformation of the fields, and propose as an ansatz that the fields only depend on space-time coordinates $x$ while the dependence on the internal coordinates $\mathbb{Y}$ enters through the group element of the transformation, $\hat{U}(\mathbb{Y})$. Since $\hat{d}$ is $O(D, D)$ invariant, it is natural to propose for it a trivial ansatz $\hat{d}(x, \mathbb{Y})=d(x)$. Instead, inspired by $(2.7)$, for the generalized metric we propose the following decomposition:

$$
\hat{\mathcal{H}}_{\hat{M} \hat{N}}(x, \mathbb{Y})=\hat{U}_{\hat{M}}^{\hat{A}}(\mathbb{Y}) \hat{\mathcal{H}}_{\hat{A} \hat{B}}(x) \hat{U}_{\hat{N}}^{\hat{B}}(\mathbb{Y}),
$$

which in terms of the vielbein reads

$$
\hat{\mathcal{E}}^{\hat{A}}{ }_{\hat{M}}(x, \mathbb{Y})=\hat{\mathcal{E}}_{\hat{B}}^{\hat{A}}(x) \hat{U}_{\hat{M}}^{\hat{B}}(\mathbb{Y})
$$

\footnotetext{
${ }^{1}$ We refer to the appendix for a discussion on the generality of considering the triangular gauge.
} 
In triangular gauge (3.7), equation (3.12) can be rewritten as

$$
\left(\begin{array}{cc}
\left(\hat{e}^{-1}\right)^{\hat{\mu}}(x, \mathbb{Y})-\left(\hat{e}^{-1}\right)^{\hat{\rho}}{ }_{\hat{m}}(x, \mathbb{Y}) \hat{B}_{\hat{\rho} \hat{\nu}}(x, \mathbb{Y}) \\
0 & \hat{e}_{\hat{\nu}}^{\hat{n}}(x, \mathbb{Y})
\end{array}\right)=\left(\begin{array}{c}
\left(\hat{e}^{-1}\right)^{\hat{p}}{ }_{\hat{m}}(x)-\left(\hat{e}^{-1}\right)^{\hat{r}}{ }_{\hat{m}}(x) \hat{B}_{\hat{r} \hat{q}}(x) \\
0 \\
\hat{e}_{\hat{q}}^{\hat{n}}(x)
\end{array}\right) \times \hat{U}(\mathbb{Y})
$$

Parameterizing the twist in terms of a diffeomorphism $\tilde{e}(\mathbb{Y})$ and $B$-transformations $\tilde{B}(\mathbb{Y})$, namely

$$
\hat{U}(\mathbb{Y})=\left(\begin{array}{cc}
\left(\tilde{e}^{-1}\right)_{\hat{p}}^{\hat{\mu}}(\mathbb{Y})-\left(\tilde{e}^{-1}\right)_{\hat{\rho}}^{\hat{\rho}}(\mathbb{Y}) \tilde{B}_{\hat{\rho} \hat{\nu}}(\mathbb{Y}) \\
0 & \tilde{e}_{\hat{\nu}}^{\hat{Q}}(\mathbb{Y})
\end{array}\right)
$$

we find that the ansatz gives the following $D$-bein and Kalb-Ramond $D$-dimensional fields

$$
\begin{aligned}
\hat{e}(x, \mathbb{Y}) & =\hat{e}(x) \tilde{e}(\mathbb{Y}) \\
\hat{B}(x, \mathbb{Y}) & =\tilde{e}^{T}(\mathbb{Y}) \hat{B}(x) \tilde{e}(\mathbb{Y})+\tilde{B}(\mathbb{Y})
\end{aligned}
$$

Following the organizing principle of [40], we impose that fields with only tangent spacetime indices are independent of the internal coordinates and this implies the following block decomposition

$$
\tilde{e}=\left(\begin{array}{ll}
1 & 0 \\
0 & e
\end{array}\right), \quad \tilde{B}=\left(\begin{array}{cc}
0 & -W^{T} \\
W & B
\end{array}\right)
$$

where $e(\mathbb{Y})$ and $B(\mathbb{Y})$ are $d \times d$ matrices and $W(\mathbb{Y})$ is a $d \times n$ matrix. By explicitly writing the dependence on the internal coordinates for the fields of the effective $n$-dimensional theory, we have

$$
\begin{aligned}
g(x, \mathbb{Y}) & =g(x), & \breve{B}(x, \mathbb{Y}) & =\breve{B}(x), \\
\mathcal{A}(x, \mathbb{Y}) & =e^{-1}(\mathbb{Y}) \mathcal{A}(x), & \breve{\mathcal{B}}(x, \mathbb{Y}) & =e^{T}(\mathbb{Y}) \breve{\mathcal{B}}(x)+W(\mathbb{Y}), \\
h(x, \mathbb{Y}) & =e^{T}(\mathbb{Y}) h(x) e(\mathbb{Y}), & b(x, \mathbb{Y}) & =e^{T}(\mathbb{Y}) b(x) e+B(\mathbb{Y}) .
\end{aligned}
$$

Note that $W$ involves space-time indices, so the ansatz produces a twist in space-time explicitly breaking Lorentz invariance. Therefore, from now on we take $W=0$.

Due to the length of the computation, we show how to obtain the effective action in two steps. In the first one, we plug the ansatz (3.11) into the action of DFT (2.4). As usual, to have simple gauge transformation properties, the reduced degrees of freedom require the following redefinitions in terms of the original higher dimensional fields

$$
\begin{aligned}
B_{\mu \nu} & =\breve{B}_{\mu \nu}-\frac{1}{2}\left(\mathcal{A}^{a}{ }_{\mu} \breve{\mathcal{B}}_{a \nu}-\mathcal{A}^{a}{ }_{\nu} \breve{\mathcal{B}}_{a \mu}\right)+\mathcal{A}^{a}{ }_{\mu} \mathcal{A}^{b}{ }_{\nu} b_{a b} \\
\mathcal{B}_{a \mu} & =\breve{\mathcal{B}}_{a \mu}-b_{a b} \mathcal{A}^{b}{ }_{\mu} .
\end{aligned}
$$


After some algebra, and using (2.6), we obtain ${ }^{2}$

$$
\begin{array}{rl}
S_{\text {eff }}=\int d^{n} & x \sqrt{|g(x)|} e^{-2 \Phi(x)}\left\{4 g^{\mu \nu}(x)\left[\partial_{\mu} \partial_{\nu} d(x)-\partial_{\mu} d(x) \partial_{\nu} d(x)\right]\right. \\
& +4 \partial_{\mu} g^{\mu \nu}(x) \partial_{\nu} d(x)-\partial_{\mu} \partial_{\nu} g^{\mu \nu}(x) \\
& +\frac{1}{8}\left[g^{\mu \nu}(x) \partial_{\mu} \hat{\mathcal{H}}^{\hat{A} \hat{B}}(x)-4 \hat{\mathcal{H}}^{\hat{A} \mu}(x) \partial_{\mu} \hat{\mathcal{H}}^{\hat{B}}(x)\right] \partial_{\nu} \hat{\mathcal{H}}_{\hat{A} \hat{B}}(x) \\
& -\frac{1}{2} F^{C}{ }_{A B} \hat{\mathcal{H}}^{B \hat{D}}(x) \hat{\mathcal{H}}^{A \mu}(x) \partial_{\mu} \hat{\mathcal{H}}_{C \hat{D}}(x) \\
& \left.-\frac{1}{4} F^{C}{ }_{D A} F^{D}{ }_{C B} \hat{\mathcal{H}}^{A B}(x)-\frac{1}{12} F^{E}{ }_{A C} F^{F}{ }_{B D} \hat{\mathcal{H}}^{A B}(x) \hat{\mathcal{H}}^{C D}(x) \hat{\mathcal{H}}_{E F}(x)\right\} .
\end{array}
$$

The dilaton $\Phi(x)$ is given by $e^{-2 \Phi(x)}=|\operatorname{det} \hat{e}(x)| \tilde{V} V_{I} e^{-2 \hat{\phi}(x)}$, where $\tilde{V}$ stands for the volume of the dual space-time and we have defined the invariant internal volume $V_{I}=$ $\int d \mathbb{Y} \operatorname{det} U=\int d \mathbb{Y}$. The field $d(x)$ on the other hand is defined by $e^{-2 d(x)}=\sqrt{|g(x)|} e^{-2 \Phi(x)}$.

The information on the twist in (3.19) becomes manifest only through the appearance of fluxes $F_{A B C}$, which are given by

$$
F_{A B C}=3 \mathcal{I}_{D[A}\left(U^{-1}\right)^{M}{ }_{B}\left(U^{-1}\right)^{N}{ }_{C} \partial_{M} U^{D}{ }_{N},
$$

where $U_{M}^{A}:=\hat{U}_{M}^{A}$. They carry $O(d, d)$ indices which are raised and lowered with the $O(d, d)$ invariant metric

$$
\mathcal{I}=\left(\begin{array}{cc}
0 & 1_{d} \\
1_{d} & 0
\end{array}\right),
$$

and, being completely antisymmetric, they belong to the $2 d(2 d-1)(2 d-2) / 3$ ! representation of $O(d, d)$. Notice that, since $U \in O(d, d)$, then $\left(U^{-1}\right)^{M}{ }_{A}=U_{A}{ }^{M}$. All the information on the twist $U(\mathbb{Y})$ in (3.14) is now encrypted in the fluxes, which must be constant in order for the effective theory to be gauge invariant. Their presence explicitly breaks $O(d, d)$ invariance and gauges a subgroup of it, as we shall see.

Next, we move to the second step where we write the action (3.19) in terms of the physical degrees of freedom (i.e. in definite representations of Lorentz and $O(d, d)$ groups). With this aim it appears convenient to specify the relation between the hatted generalized metric and the physical fields. Namely

$$
\begin{aligned}
& \hat{\mathcal{H}}^{A B}(x)=\mathcal{H}^{A B}+A^{A}{ }_{\mu} g^{\mu \nu} A^{B}{ }_{\nu}, \\
& \hat{\mathcal{H}}^{A \nu}(x)=-A^{A}{ }_{\mu} g^{\mu \nu}, \\
& \hat{\mathcal{H}}_{A \nu}(x)=\mathcal{H}_{A B} A^{B}{ }_{\nu}+A_{A \rho} g^{\rho \mu}\left(B_{\mu \nu}+\frac{1}{2} A_{\mu}^{B} A_{B \nu}\right), \\
& \hat{\mathcal{H}}^{\mu \nu}(x)=g^{\mu \nu}, \\
& \hat{\mathcal{H}}^{\mu}{ }_{\nu}(x)=-g^{\mu \rho}\left(B_{\rho \nu}+\frac{1}{2} A_{\rho}^{B} A_{B \nu}\right) \\
& \hat{\mathcal{H}}_{\mu \nu}(x)=g_{\mu \nu}+A^{A}{ }_{\mu} \mathcal{H}_{A B} A^{B}{ }_{\nu}+\left(B_{\rho \mu}+\frac{1}{2} A_{\rho}^{B} A_{B \mu}\right) g^{\rho \sigma}\left(B_{\sigma \nu}+\frac{1}{2} A_{\sigma}^{B} A_{B \nu}\right)
\end{aligned}
$$

\footnotetext{
${ }^{2}$ Since the twist matrix is not necessarily globally well defined, gauge invariance of the original action (2.4) requires the additional constraint $\partial_{M}\left(U^{-1}\right)^{M}{ }_{A}=0$, which we assume from now on.
} 
where the physical degrees of freedom are encoded as:

- Scalars are arranged in the $O(d, d)$ covariant scalar matrix

$$
\mathcal{H}_{A B}=\left(\begin{array}{cc}
h^{a b} & -h^{a c} b_{c b} \\
b_{a c} h^{c b} & h_{a b}-b_{a c} h^{c d} b_{d b}
\end{array}\right) .
$$

There are $d(d+1) / 2+d(d-1) / 2$ real scalars in total, each coming from the internal components of the $D$-dimensional metric and $B$-field, respectively. In addition there is also the dilaton $\Phi$.

- Vectors are formed by the $d+d$ vectorial degrees of freedom coming from the metric and $B$-field, respectively (see (3.10) and (3.18)). They are arranged in a fundamental representation of $O(d, d)$ as

$$
A_{\mu}^{A}=\left(\begin{array}{c}
\mathcal{B}_{a \mu} \\
-\mathcal{A}^{a}{ }_{\mu}
\end{array}\right) .
$$

- Tensors are given by the $n$-dimensional 2 -form field $B_{\mu \nu}$ and the $n$-dimensional metric $g_{\mu \nu}$.

After some algebra, the effective action can be finally written in a standard form as (see the set of equations (3.22))

$$
\begin{aligned}
S_{\text {eff }}=\int d^{n} x \sqrt{|g(x)|} e^{-2 \Phi(x)} & \left\{\mathbf{R}+4 \partial^{\mu} \Phi \partial_{\mu} \Phi-\frac{1}{4} \mathcal{H}_{A B} \mathcal{F}^{A \mu \nu} \mathcal{F}^{B}{ }_{\mu \nu}\right. \\
& -\frac{1}{12} g^{\mu \nu} g^{\rho \sigma} g^{\lambda \tau} \mathcal{G}_{\mu \rho \lambda} \mathcal{G}_{\nu \sigma \tau}+\frac{1}{8} D_{\mu} \mathcal{H}_{A B} D^{\mu} \mathcal{H}^{A B} \\
& \left.-\frac{1}{4} F^{C}{ }_{D A} F^{D}{ }_{C B} \mathcal{H}^{A B}-\frac{1}{12} F^{E}{ }_{A C} F^{F}{ }_{B D} \mathcal{H}^{A B} \mathcal{H}^{C D} \mathcal{H}_{E F}\right\} .
\end{aligned}
$$

Here $\mathbf{R}$ is the $n$-dimensional Ricci scalar, and we have defined the field strengths as

$$
\begin{aligned}
\mathcal{F}^{A}{ }_{\mu \nu} & =\partial_{\mu} A^{A}{ }_{\nu}-\partial_{\nu} A^{A}{ }_{\mu}-F^{A}{ }_{B C} A^{B}{ }_{\mu} A^{C}{ }_{\nu}, \\
\mathcal{G}_{\mu \rho \lambda} & =3 \partial_{[\mu} B_{\rho \lambda]}-F_{A B C} A^{A}{ }_{\mu} A^{B}{ }_{\rho} A^{C}{ }_{\lambda}+3 \partial_{[\mu} A^{A}{ }_{\rho} A_{\lambda] A},
\end{aligned}
$$

and a covariant derivative for scalars as

$$
D_{\mu} \mathcal{H}_{A B}=\partial_{\mu} \mathcal{H}_{A B}-F^{C}{ }_{A D} A^{D}{ }_{\mu} \mathcal{H}_{C B}-F^{C}{ }_{B D} A^{D}{ }_{\mu} \mathcal{H}_{A C} .
$$

The effective theory (3.25) has remnant symmetries that are inherited from the global symmetry (2.7) and the gauge invariance (2.8) of its parent DFT. Concerning the former, although the effective theory is written in a manifestly covariant $O(d, d)$ form, the fluxes explicitly break $O(d, d)$ invariance, which is only recovered if they are treated as spurionic fields. With respect to gauge invariance, given a $2 D$-dimensional gauge parameter $\hat{\xi}^{\hat{M}}$, the gauge transformations (2.8) can be twisted as

$$
\hat{\xi}^{\hat{M}}(x, \mathbb{Y})=\left(\hat{U}^{-1}(\mathbb{Y})\right)_{\hat{A}}^{\hat{M}} \xi^{\hat{A}}(x),
$$


and, when evaluated on the C-bracket (2.11), the following effective algebra is induced

$$
\left[\delta_{\xi_{1}}, \delta_{\xi_{2}}\right]_{C}=\delta_{\xi_{3}}, \quad \xi_{3}^{A}=-F_{B C}^{A} \xi_{2}^{B} \xi_{1}^{C},
$$

where the structure constants are given by the fluxes (3.20). In terms of the gauge parameter $\xi^{A}(x)$ the fields transform as

$$
\begin{aligned}
\delta_{\xi} g_{\mu \nu}(x) & =0 \\
\delta_{\xi} d(x) & =0 \\
\delta_{\xi} B_{\mu \nu}(x) & =-\frac{1}{2}\left(A^{A}{ }_{\mu} \partial_{\nu} \xi_{A}-A^{A}{ }_{\nu} \partial_{\mu} \xi_{A}\right), \\
\delta_{\xi} A^{A}{ }_{\mu}(x) & =-\partial_{\mu} \xi^{A}(x)-F^{A}{ }_{B C} \xi^{B}(x) A^{C}{ }_{\mu}, \\
\delta_{\xi} \mathcal{H}_{A B}(x) & =-F_{A C}{ }^{D} \xi^{C}(x) \mathcal{H}_{D B}(x)-F_{B C}{ }^{C} \xi^{C}(x) \mathcal{H}_{A D}(x) .
\end{aligned}
$$

The twists define the infinitesimal generators of this symmetry group, namely

$$
\mathcal{E}_{A}=\left(U^{-1}\right)_{A}{ }^{P} \mathcal{E}_{P},
$$

which satisfy the following C-algebra

$$
\left[\mathcal{E}_{A}, \mathcal{E}_{B}\right]_{C}=F^{D}{ }_{A B} \mathcal{E}_{D} .
$$

The Jacobiator associated to the C-bracket does not automatically vanish, so it is not $a$ priori evident whether the fluxes satisfy Jacobi identities or not. To answer this question we note that the C-bracket differs from the D-bracket (which, although non skew-symmetric, does satisfy Jacobi) by a total derivative [28]

$$
[A, B]_{D}^{R}=[A, B]_{C}^{R}+\frac{1}{2} \partial^{R}\left(A^{N} B_{N}\right) .
$$

When particularly evaluated on the twists $U(\mathbb{Y})$, the last term vanishes

$$
\partial^{R}\left(\left(U^{-1}\right)^{M}{ }_{A} \mathcal{I}_{M N}\left(U^{-1}\right)^{N}{ }_{B}\right)=\partial^{R} \mathcal{I}_{A B}=0,
$$

restoring anti-symmetry and leading to a vanishing Jacobiator. We thus conclude that the fluxes must be constrained by the usual Jacobi identities

$$
F_{[A B}^{C} F_{D] C}^{E}=0 .
$$

\section{The effective action of DFT and gauged $\mathcal{N}=4$ supergravity}

Setting $n=4$ and $d=6$, the effective action of DFT derived in the previous section reduces to a four dimensional gauge theory with an $O(6,6)$ global invariance. Interestingly enough, $O(6,6)$ is the symmetry group of four dimensional gauged $\mathcal{N}=4$ supergravity. Therefore, even if the starting DFT does not include supersymmetry, we could expect a connection with the bosonic sector of gauged supergravity. Indeed, in this section we show that the effective action of DFT reproduces the electric bosonic sector of four dimensional gauged $\mathcal{N}=4$ supergravity.

We begin by reviewing this theory in the formulation of Schon and Weidner [42] and then establish the precise correspondence. The notation used in [42] differs from ours, and the relation between both conventions is specified in table 1 and in equation (4.13) below. 


\subsection{Review of gauged $\mathcal{N}=4$ supergravity}

The 4-dimensional ungauged $\mathcal{N}=4$ supergravity has a global symmetry $G=S L(2) \times$ $O(6,6+N)$, whose maximal compact subgroup $K=U(1) \times O(6) \times O(6+N)$ is realized on-shell as a gauge symmetry of the theory. The part of its field content that will be relevant for our discussion is arranged in a gravity multiplet and $6+N$ vector multiplets involving the following fields:

- The gravity multiplet contains the metric $g_{\mu \nu}$, six vectors $B_{m \mu}(m=1, \ldots, 6)$ and one complex scalar $\tau$ parameterizing the coset $S U(2) / U(1)$. It is useful to define the $K$ invariant matrix

$$
M_{\alpha \beta}=\frac{1}{\operatorname{Im} \tau}\left(\begin{array}{cc}
|\tau|^{2} & \operatorname{Re} \tau \\
\operatorname{Re} \tau & 1
\end{array}\right)
$$

with $S L(2)$ indices $\alpha, \beta= \pm$. In addition, it contains four gravitinos $\psi_{\mu}^{i}$ and four spin- $1 / 2$ fermions $\psi^{i}(i=1, \ldots, 4)$. The fermions are singlets under $G$ and rotate among each other through $S U(4) \approx O(6) \in K$.

- Each vector multiplet contains one vector $A_{\mu}^{a}$ (a labels the vector multiplet), six real scalars and four spin-1/2 gauginos $\lambda_{a}^{i}$. Again, the index $i=1, \ldots, 4$ is a gauge index rotated by $O(6) \in K$, and the vector multiplets are rotated among each other by $O(6+N) \in K$. All the $6 \times(6+N)$ real scalars parameterize the coset

$$
\mathcal{V}_{M}^{A} \in \frac{O(6,6+N)}{O(6) \times O(6+N)},
$$

with $M$ an $O(6,6+N)$ index and $A$ splits into $(m, a)$ with $m$ an $O(6)$ index and $a$ an $O(6+N)$ index. This vielbein is an element of $O(6,6+N)$ so it satisfies

$$
\eta_{M N}=-\mathcal{V}_{M}^{m} \mathcal{V}_{N}^{m}+\mathcal{V}_{M}{ }^{a} \mathcal{V}_{N}{ }^{a}, \quad \eta_{M N}=\operatorname{diag}(------,+\ldots+)
$$

As for $\tau$, one can construct a $K$-invariant scalar matrix

$$
M_{M N}=\mathcal{V}_{M}^{a} \mathcal{V}_{N}^{a}+\mathcal{V}_{M}^{m} \mathcal{V}_{M}^{m}
$$

Finally, the 6 vectors of the gravity multiplet together with the $6+N$ vectors of the vector multiplets combine to form the electric vector field in the fundamental of $O(6,6+N)$

$$
A_{\mu}{ }^{M+}=\left(\begin{array}{c}
B_{m \mu} \\
A_{\mu}^{a}
\end{array}\right) .
$$

In the description of [42], the ungauged theory contains the metric $g_{\mu \nu}$, electric vector fields $A_{\mu}{ }^{M+}$ and scalars $\tau$ and $M_{M N}$ as free fields in the Lagrangian, while the dual magnetic vectors $A_{\mu}{ }^{M-}$ and two-form gauge fields $B_{\mu \nu}^{M N}$ and $B_{\mu \nu}^{\alpha \beta}$ are only introduced on-shell. The gauging of the global group is parameterized by the embedding tensors in the following representations of $G$

$$
f_{\alpha M N P} \in(\mathbf{2}, \mathbf{2 2 0}), \quad \xi_{\alpha M} \in(\mathbf{2}, \mathbf{1 2}) .
$$


When these gaugings are turned on, some of the fields that were absent off-shell in the ungauged theory are now present.

The bosonic Lagrangian is a sum of three terms $\mathcal{L}_{\text {bos }}=\mathcal{L}_{\text {kin }}+\mathcal{L}_{\text {pot }}+\mathcal{L}_{\text {top }}$ given by

$$
\begin{gathered}
e^{-1} \mathcal{L}_{\text {kin }}=\frac{1}{2} \mathbf{R}+\frac{1}{16} D_{\mu} M_{M N} D^{\mu} M^{M N}+\frac{1}{8} D_{\mu} M_{\alpha \beta} D^{\mu} M^{\alpha \beta} \\
-\frac{1}{4} \operatorname{Im}(\tau) M_{M N} \mathcal{H}_{\mu \nu}^{M+} \mathcal{H}^{\mu \nu N+}+\frac{1}{8} \operatorname{Re}(\tau) \eta_{M N} \epsilon^{\mu \nu \rho \lambda} \mathcal{H}_{\mu \nu}^{M+} \mathcal{H}_{\rho \lambda}^{N+} \\
e^{-1} \mathcal{L}_{\text {pot }}=-\frac{g^{2}}{16}\left\{f_{+M N P} f_{+Q R S} M^{++}\left[\frac{1}{3} M^{M Q} M^{N R} M^{P S}+\left(\frac{2}{3} \eta^{M Q}-M^{M Q}\right) \eta^{N R} \eta^{P S}\right]\right. \\
\left.+3 \xi_{+}^{M} \xi_{+}^{N} M^{++} M_{M N}\right\}+\ldots, \\
e^{-1} \mathcal{L}_{\text {top }}=-\frac{g}{2} \epsilon^{\mu \nu \rho \lambda}\left\{\begin{array}{r}
\xi_{+M} \eta_{N P} A_{\mu}^{M-} A_{\nu}^{N+} A_{\lambda}^{P+}-\frac{g}{4} \hat{f}_{+M N R} \hat{f}_{+P Q}{ }^{R} A_{\mu}^{M+} A_{\nu}^{N+} A_{\rho}^{P+} A_{\lambda}^{Q-} \\
\left.-\frac{1}{4} \xi_{+M} B_{\mu \nu}^{++}\left(2 \partial_{\rho} A_{\lambda}^{M-}-g \hat{f}_{+Q R}{ }^{M} A_{\rho}^{Q+} A_{\lambda}^{R-}\right)\right\}+\ldots,
\end{array}\right.
\end{gathered}
$$

where the dots involve terms containing magnetic gaugings $f_{-M N P}$ and $\xi_{-M}$. The covariant derivatives are defined by

$$
\begin{aligned}
D_{\mu} M_{\alpha \beta} & =\partial_{\mu} M_{\alpha \beta}+g A_{\mu}^{M \gamma} \xi_{(\alpha M} M_{\beta) \gamma}-g A_{\mu}^{M \delta} \xi_{+M} \epsilon_{\delta(\alpha} M_{\beta)-}+\ldots \\
D_{\mu} M_{M N} & =\partial_{\mu} M_{M N}+2 g A_{\mu}^{P+} \Theta_{+P(M} M_{N) Q}+\ldots
\end{aligned}
$$

the field strength by

$$
\mathcal{H}_{\mu \nu}^{M+}=2 \partial_{[\mu} A_{\nu]}^{M+}-g \hat{f}_{+N P}^{M} A_{[\mu}^{N \alpha} A_{\nu]}^{P+}+\frac{g}{2} \xi_{+}^{M} B_{\mu \nu}^{++}+\ldots,
$$

and also the following combinations of gaugings were defined

$$
\Theta_{+M N P}=f_{+M N P}-\xi_{+[N} \eta_{P] M}, \quad \hat{f}_{+M N P}=f_{+M N P}-\xi_{+[M} \eta_{P] N}-\frac{3}{2} \xi_{+N} \eta_{M P} .
$$

Finally, we mention that the gaugings must satisfy the following consistency quadratic constraints

$$
\begin{aligned}
\xi_{+}^{M} \xi_{+M} & =0, \\
\xi_{+}^{P} f_{+P M N} & =0, \\
3 f_{+R[M N} f_{+P Q]}+2 \xi_{+[M} f_{+N P Q]} & =0, \quad \ldots
\end{aligned}
$$

\subsection{Comparison with the effective action of DFT}

Having reviewed the on-shell formulation of $\mathcal{N}=4$ gauged supergravity, let us proceed to compare it with (3.25) in the particular case $n=4, d=6$. First we note that since the effective action of DFT has no $S L(2)$ symmetry mixing electric with magnetic sectors, we can only hope to reproduce one of these sectors, which we take to be the electric one. Since the vectors in the effective action of DFT arise only from the metric and B-field, we must set $N=0$. We then show how to relax this restriction and finally, by gauging the rescaling symmetry, we propose a way to obtain the gaugings $\xi_{+M}$. 


\begin{tabular}{|c|c|}
\hline Effective DFT action & Gauged $\mathcal{N}=4$ sugra \\
\hline$F_{A B C}$ & $g f_{+M N P}$ \\
$\mathcal{H}_{A B}$ & $M_{M N}$ \\
$e^{-2 \Phi}$ & $2 \operatorname{Im}(\tau)$ \\
$A_{\mu}^{A}$ & $A_{\mu}^{M+}$ \\
\hline
\end{tabular}

Table 1. Correspondence between our conventions and those in [42].

Note that the $O(6,6)$ metrics employed in (3.21) and (4.2) differ, the relation between them being

$$
R^{T} \eta R=\mathcal{I}, \quad R=\frac{1}{\sqrt{2}}\left(\begin{array}{cc}
1 & -1 \\
1 & 1
\end{array}\right) \in S O(12) .
$$

Therefore, all the comparisons made in this section are only valid up to a rotation by this matrix. We implicitly assume this fact.

\subsubsection{The $O(6,6)$ sector}

The fluxes in (3.20) belong to the $\mathbf{2 2 0}$ of $O(6,6)$. It is then natural to identify them with the electric gaugings and we must then turn off all the magnetic fluxes $f_{-M N P}$ and $\xi_{-M}$. There is no other source of deformations so far, so we must also set $\xi_{+M}=0$ (the inclusion of the full set of electric gaugings is discussed in the forthcoming subsections).

To perform the comparison, the effective action of DFT (3.25) must first be taken to the Einstein frame. The transformation $g_{\mu \nu} \rightarrow e^{2 \Phi} g_{\mu \nu}$ gives

$$
\begin{aligned}
S_{\text {eff }}=\int d^{4} x \sqrt{|g(x)|} & \left\{\frac{1}{2} \mathbf{R}+\frac{1}{16} D_{\mu} \mathcal{H}_{A B} D^{\mu} \mathcal{H}^{A B}-\frac{1}{8} e^{-2 \Phi} \mathcal{H}_{A B} \mathcal{F}^{A \mu \nu} \mathcal{F}^{B}{ }_{\mu \nu}\right. \\
- & \partial^{\mu} \Phi \partial_{\mu} \Phi-\frac{1}{24} g^{\mu \nu} g^{\rho \sigma} g^{\lambda \tau} e^{-4 \Phi} \mathcal{G}_{\mu \rho \lambda} \mathcal{G}_{\nu \sigma \tau} \\
- & \left.e^{2 \Phi} \frac{1}{8} F^{C}{ }_{D A} F^{D}{ }_{C B} \mathcal{H}^{A B}-e^{2 \Phi} \frac{1}{24} F^{E}{ }_{A C} F^{F}{ }_{B D} \mathcal{H}^{A B} \mathcal{H}^{C D} \mathcal{H}_{E F}\right\} .
\end{aligned}
$$

Then one should make the identifications in table 1 and this guarantees that both actions and also their respective constraints coincide. The following subtleties must be taken into account when performing the comparison:

1. Due to quadratic constraints (4.12), when $f_{-M N P}=\xi_{-M}=\xi_{+M}=0$, the topological terms vanish $\mathcal{L}_{\text {top }}=0$. To see this, note that

$$
f_{+R[M N} f_{+P Q]}^{R}=0 \quad \Leftrightarrow \quad f_{+R[M N} f_{+P] Q}^{R}=0 .
$$

2. The term $g^{2} f_{+M N P} f^{+M N P}$ in $\mathcal{L}_{\text {pot }}$ can be explicitly written in terms of the twist, by using (3.20), as

$$
\begin{aligned}
\frac{1}{3} F_{A B C} F^{A B C} & =\partial_{M} U^{A}{ }_{N} \partial^{M}\left(U^{-1}\right)^{N}{ }_{A} \\
& =-\frac{1}{2}\left(\partial_{M} \partial^{M} U^{A}{ }_{N}\right)\left(U^{-1}\right)^{N}{ }_{A}-\frac{1}{2} U^{A}{ }_{N}\left(\partial_{M} \partial^{M}\left(U^{-1}\right)^{N}{ }_{A}\right)
\end{aligned}
$$


and therefore it vanishes due to the level matching condition. This in turn implies that the effective action (4.14) is in fact a truncation of an $\mathcal{N}=8$ theory to $\mathcal{N}=4[44,45]$, so only a subset of gauged $\mathcal{N}=4$ supergravities can be reached through ScherkSchwarz compactification from DFT.

3. To make the equivalence evident, the two-form $B_{\mu \nu}$ must be dualized in the effective action of DFT to give an equivalent theory in terms of a scalar field $\lambda \equiv 2 \operatorname{Re}(\tau)$. Introducing $\lambda$ as a Lagrange multiplier to enforce $\epsilon^{\sigma \mu \nu \rho} \partial_{\sigma} \partial_{\mu} B_{\nu \rho}=0$, one defines the following action for $B_{\mu \nu}$

$$
S_{B}=-\int \frac{d^{4} x}{24} \sqrt{g}\left[e^{-4 \Phi} \mathcal{G}_{\mu \nu \rho} \mathcal{G}^{\mu \nu \rho}+2 \lambda \epsilon^{\sigma \mu \nu \rho} \partial_{\sigma}\left(\mathcal{G}_{\mu \nu \rho}+F_{A B C} A_{\mu}^{A} A_{\nu}^{B} A_{\rho}^{C}-3 \partial_{\mu} A_{\nu}^{A} A_{A \rho}\right)\right] .
$$

Here $\mathcal{G}_{\mu \nu \rho}$ must be eliminated through its equation of motion

$$
\mathcal{G}^{\mu \nu \rho}=e^{4 \Phi} \epsilon^{\sigma \mu \nu \rho} \partial_{\sigma} \lambda
$$

which replaced in $S_{B}$ gives

$$
S_{B}=-\int \frac{d^{4} x}{24} \sqrt{g} e^{4 \Phi}\left[6 \partial_{\sigma} \lambda \partial^{\sigma} \lambda-2 \lambda \epsilon^{\sigma \mu \nu \rho} \partial_{\sigma}\left(-F_{A B C} A_{\mu}^{A} A_{\nu}^{B} A_{\rho}^{C}+3 \partial_{[\mu} A_{\nu}^{A} A_{\rho] A}\right)\right] .
$$

The first term contributes to the kinetic term for the gravity scalars, and the second one coincides with the last term in $\mathcal{L}_{\text {kin }}$ upon use of quadratic constraints.

\subsubsection{Including vectors}

We showed above that the effective action of DFT matches the bosonic electric sector of gauged $\mathcal{N}=4$ supergravity when the number of vector multiplets is six, i.e. $N=0$, and $\xi_{+M}=0$. Here we show how to relax the first restriction.

To increase the number of electric vector fields, we are inspired by the extension of DFT to the abelian heterotic string performed in [30], where the $2 D$ coordinates $X^{\hat{\mu}}, \tilde{X}_{\hat{\mu}}, \hat{\mu}=$ $0, \ldots, D-1$ are extended by $N$ extra coordinates $z^{i}, i=1, \ldots, N$ and, correspondingly, the generalized metric $\hat{\mathcal{H}}_{\hat{M} \hat{N}}$ is enlarged to a $(2 D+N) \times(2 D+N)$ matrix that naturally incorporates $N$ additional vector fields $\hat{V}_{\hat{\mu}}^{i}$.

We will use the following $O(D, D+N)$ metric

$$
\hat{\mathcal{I}}_{\hat{M} \hat{N}}=\left(\begin{array}{ccc}
\mathcal{I}^{\hat{\mu} \hat{\nu}} & \mathcal{I}^{\hat{\mu}}{ }_{\hat{\nu}} \mathcal{I}^{\hat{\mu}}{ }_{i} \\
\mathcal{I}_{\hat{\mu}}^{\hat{\nu}} & \mathcal{I}_{\hat{\mu} \hat{\nu}} & \mathcal{I}_{\hat{\mu} i} \\
\mathcal{I}_{i}^{\hat{\nu}} & \mathcal{I}_{i \hat{\nu}} & \mathcal{I}_{i j}
\end{array}\right)=\left(\begin{array}{ccc}
0 & 1_{D} & 0 \\
1_{D} & 0 & 0 \\
0 & 0 & 1_{N}
\end{array}\right)
$$

We formally keep the action and the form of the gauge transformations but with respect to the enlarged generalized metric $\hat{\mathcal{H}}_{\hat{M} \hat{N}}$ introduced in [30], namely

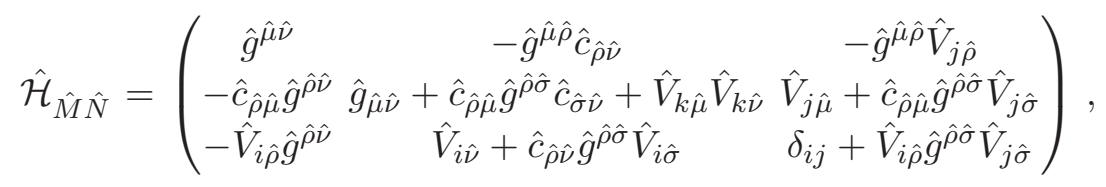


where the indices of the gauge group $i, j, \ldots$ are raised and lowered with $\delta_{i j}$ and

$$
\hat{c}_{\hat{\mu} \hat{\nu}}=\hat{B}_{\hat{\mu} \hat{\nu}}+\frac{1}{2} \hat{V}_{\hat{\mu}}^{i} \hat{V}_{i \hat{\nu}}
$$

The generalized vielbein producing $\hat{\mathcal{H}}_{\hat{M} \hat{N}}=\mathcal{E}^{\hat{A}} \hat{M}_{\hat{M}} \hat{\eta}_{\hat{A} \hat{B}} \hat{\mathcal{B}}^{\hat{B}}{ }_{\hat{N}}$ is

$$
\hat{\mathcal{E}}_{\hat{N}}^{\hat{B}}=\left(\begin{array}{ccc}
\left(\hat{e}^{-1}\right)^{\hat{\nu}} \hat{n}-\left(\hat{e}^{-1}\right)^{\hat{\sigma}} \hat{n}_{\hat{n} \hat{\sigma} \hat{\nu}}-\left(\hat{e}^{-1}\right)^{\hat{\sigma}_{\hat{n}}} \hat{V}_{\hat{\sigma} j} \\
0 & \hat{e}^{\hat{n}} \hat{\nu} & 0 \\
0 & \hat{V}_{i \hat{\nu}} & \delta_{i j}
\end{array}\right) .
$$

The compactification procedure closely follows [41]. The metric and $B$ fields in terms of the compactified degrees of freedom take the form (3.10), where now

$$
\begin{aligned}
B_{\mu \nu} & =\breve{B}_{\mu \nu}-\frac{1}{2}\left(\mathcal{A}_{\mu}^{a} B_{a \nu}-\mathcal{A}_{\nu}^{a} B_{a \mu}\right)-b_{a b} \mathcal{A}_{\mu}^{a} \mathcal{A}_{\nu}^{b}-\frac{1}{2} \hat{V}_{c}^{i}\left(\mathcal{A}_{\mu}^{c} V_{\nu}^{i}-\mathcal{A}_{\nu}^{c} V_{\mu}^{i}\right), \\
\mathcal{B}_{a \mu} & =\breve{\mathcal{B}}_{a \mu}-b_{a c} \mathcal{A}_{\mu}^{c}-\frac{1}{2} V_{a}^{i} V_{\mu}^{i},
\end{aligned}
$$

and the vector fields are

$$
V_{\mu}^{i}=\hat{V}_{\mu}^{i}-\hat{V}_{c}^{i} \mathcal{A}_{\mu}^{c} .
$$

Generalizing the procedure of the previous section, we define the reduced gauge multiplet as

$$
A_{\mu}^{A}=\left(\begin{array}{c}
\mathcal{B}_{a \mu} \\
-\mathcal{A}_{\mu}^{a} \\
-V_{\mu}^{i}
\end{array}\right)
$$

and the reduced generalized metric as

$$
\mathcal{H}_{A B}=\left(\begin{array}{ccc}
h^{a b} & -h^{a d} c_{d b} & -h^{a d} V_{\gamma}^{j} \\
c_{a d} h^{d b} & h_{a b}-c_{a d} h^{d e} c_{e b}+V_{a}^{i} V_{b}^{i} & V_{a}^{j}+c_{d a} h^{d e} V_{e}^{j} \\
-V_{d}^{i} h^{b d} & V_{b}^{i}+c_{d b} h^{d e} V_{\delta}^{i} & \delta_{i j}+V_{d}^{i} h^{d e} V_{e}^{j}
\end{array}\right),
$$

where $c_{a b}=b_{a b}+\frac{1}{2} V_{a}^{i} V_{b}^{i}$.

The global symmetry to be twisted is now $O(D, D+N)$ and we extend the ansatz (3.11) for the generalized metric as

$$
\hat{\mathcal{H}}_{\hat{M} \hat{N}}(x, \mathbb{Z})=\hat{U}_{\hat{M}}^{\hat{A}}(\mathbb{Z}) \hat{\mathcal{H}}_{\hat{A} \hat{B}}(x) \hat{U}_{\hat{N}}^{\hat{B}}(\mathbb{Z}),
$$

where $\mathbb{Z}=(\mathbb{Y}, z)$ and $\hat{U} \in O(D, D+N)$. In terms of the vielbein, it reads

$$
\hat{\mathcal{E}}^{\hat{A}}{ }_{\hat{M}}(x, \mathbb{Z})=\hat{\mathcal{E}}^{\hat{A}}{ }_{\hat{B}}(x) \hat{U}_{\hat{M}}^{\hat{B}}(\mathbb{Z}),
$$

which can be rewritten in matricial form as

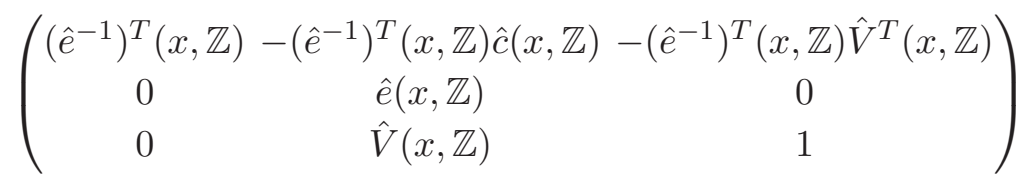

$$
\begin{aligned}
& =\left(\begin{array}{ccc}
\left(\hat{e}^{-1}\right)^{T}(x) & -\left(\hat{e}^{-1}\right)^{T}(x) \hat{c}(x) & -\left(\hat{e}^{-1}\right)^{T}(x) \hat{V}^{T}(x) \\
0 & \hat{e}(x) & 0 \\
0 & \hat{V}(x) & 1
\end{array}\right) \times \hat{U}(\mathbb{Z}) .
\end{aligned}
$$


Parameterizing the twist in terms of diffeomorphisms $\tilde{e}, \tilde{B}$ and $\tilde{V}$ transformations, namely

$$
\hat{U}(\mathbb{Z})=\left(\begin{array}{ccc}
\left(\tilde{e}^{-1}\right)^{T}-\left(\tilde{e}^{-1}\right)^{T} & \tilde{c}-\left(\tilde{e}^{-1}\right)^{T} \tilde{V}^{T} \\
0 & \tilde{e} & 0 \\
0 & \tilde{V} & 1
\end{array}\right),
$$

the ansatz gives the following $D$-dimensional gauge fields

$$
\hat{V}(x, \mathbb{Z})=\tilde{V}(\mathbb{Z})+\tilde{e}(\mathbb{Z}) \hat{V}(x) .
$$

Now everything goes on exactly as before. The reduced effective action has the same form of the previous section, namely (3.25), with the obvious extension in the values taken by the indices and the inclusion of $N$ extra vector fields $V_{\mu}^{i}$ in the definition (4.24).

\subsubsection{Rescaling symmetry gaugings}

In this section we propose a way to obtain the gaugings $\xi_{+M}$ in the $(\mathbf{2}, \mathbf{1 2})$ representations of $S L(2) \times O(6,6)$. For simplicity here we restrict to the $N=0$ case, but the results can be extended if heterotic vector multiplets are introduced as in the previous subsection and their rescaling taken into account. To facilitate the presentation, in this subsection we turn off the gaugings in the $(\mathbf{2}, \mathbf{2 2 0})$.

In [46], a rescaling symmetry of the NS-NS sector of the heterotic theory was twisted, and it was shown that this gives rise to the gaugings $\xi_{+m}$ with $m=1, \ldots 6$. This symmetry acts by rescaling the metric and $B$-field, and by shifting the dilaton, and it is explicitly broken in DFT due to the dependence on the dual coordinates. However, at a formal level DFT is invariant under a global symmetry given by

$$
\hat{d} \rightarrow \hat{d} \pm \gamma, \quad \hat{\mathcal{H}}^{\hat{M} \hat{N}} \rightarrow \hat{O}^{\hat{M}}{ }_{\hat{P}} \hat{\mathcal{H}}^{\hat{P} \hat{Q}} \hat{O}^{\hat{N}}{ }_{\hat{Q}}, \quad \hat{O}=\left(\begin{array}{cc}
e^{\gamma} & 0 \\
0 & e^{-\gamma}
\end{array}\right)
$$

whenever the following relation holds

$$
\hat{O}_{\hat{M}}^{\hat{P}} \partial_{\hat{P}} \rightarrow e^{ \pm \gamma} \partial_{\hat{M}},
$$

i.e., when either

$$
\partial^{m} \bullet=0 \quad(-)
$$

or

$$
\partial_{m} \bullet=0 \quad(+) .
$$

While the former (4.33) corresponds to the case analyzed in [46], the later is only possible due to the doubling of the coordinates, and symmetry arguments suggest that they should give rise to the $\xi_{+}^{m}$ gaugings. Here we use the covariant notation (4.31), but one should keep in mind that the extension of the symmetry is only possible if covariance is broken through either (4.33) or (4.34).

The compactification procedure is equivalent to the one before. One transforms the fields and makes the transformations carry the internal dependence

$$
\hat{d}(x, \mathbb{Y})=\hat{d}(x) \pm \gamma(\mathbb{Y}), \quad \hat{\mathcal{H}}^{\hat{M} \hat{N}}(x, \mathbb{Y}) \rightarrow \hat{O}^{\hat{M}}{ }_{\hat{P}}(\mathbb{Y}) \hat{\mathcal{H}}^{\hat{P}} \hat{Q}_{(x)} \hat{O}^{\hat{N}}{ }_{\hat{Q}}(\mathbb{Y}) .
$$


The \pm depends on whether (4.33) or (4.34) is chosen.

The comparison of the effective action with gauged $\mathcal{N}=4$ supergravity is not straightforward, and one must use the dualizations of [46]. Instead of dualizing the $B$-field into the axion of [42], one must integrate the $A_{\mu}^{M-}$ fields out and use the local gauge invariance under (axionic) rescaling shifts to gauge away the axion $\operatorname{Im} \tau=0$. This yields the following equivalent formulation of gauged $\mathcal{N}=4$ supergravity when only $\xi_{+}$gaugings are turned on

$$
\begin{aligned}
e^{-1} \mathcal{L}= & \frac{1}{2} R+\frac{1}{16} D_{\mu} M_{M N} D^{\mu} M^{M N}-D_{\mu} \phi D^{\mu} \phi \\
& -\frac{1}{4} e^{-2 \phi} M_{M N} \mathcal{H}_{\mu \nu}^{M+} \mathcal{H}^{N \mu \nu+}-\frac{3}{8} e^{-4 \phi} g^{2} \Omega_{\mu \nu \rho} \Omega^{\mu \nu \rho}+e^{-1} \mathcal{L}_{p o t},
\end{aligned}
$$

where

$$
\begin{aligned}
D_{\mu} \phi & =\partial_{\mu} \phi-\frac{1}{2} \xi_{+M} A_{\mu}^{M+} \\
D_{\mu} M_{M N} & =\partial_{\mu} M_{M N}-g A_{\mu}^{P+} \xi_{+M} M_{N P}+g A_{\mu M}^{+} M_{N P} \xi^{+P} \\
\Omega_{\mu \nu \rho} & =\partial_{[\mu} B_{\nu \rho]}^{++}-\xi_{+M} A_{[\mu}^{M+} B_{\nu \rho]}^{++}-2 A_{[\mu}^{N+} \partial_{\nu} A_{\rho] N}^{+} \\
\mathcal{H}_{\mu \nu}^{M+} & =2 \partial_{[\mu} A_{\nu]}^{M+}-\frac{g}{2} \xi_{+N} A_{[\mu}^{N+} A_{\nu]}^{M+}+\frac{g}{2} \xi_{+}^{M} B_{\mu \nu}^{++} .
\end{aligned}
$$

The identifications are those of the previous section plus $B_{\mu \nu}^{++} \leftrightarrow B_{\mu \nu}$ and the gaugings are originated from the following derivatives

$$
\xi_{+M} \leftrightarrow \partial_{M} \gamma(\mathbb{Y})
$$

Equations (4.33)-(4.34) imply that the constraints $\xi_{+}^{M} \xi_{+M}=0$ are automatically satisfied.

\section{5 (Non)geometric string backgrounds}

In this section we show that the generalized Scherk-Schwarz mechanism can be interpreted as a reduction on a twisted double torus. The string backgrounds associated to different twists are analyzed and their connection to standard (dual) fluxes is discussed.

\subsection{Reduction on a twisted double torus}

The dependence of the generalized metric and its vielbein on the internal coordinates $\mathbb{Y}^{M}$ was introduced in (3.11) as

$$
\mathcal{H}_{M N}(x, \mathbb{Y})=U^{A}{ }_{M}(\mathbb{Y}) \mathcal{H}_{A B}(x) U^{B}{ }_{N}(\mathbb{Y}) .
$$

In the limit in which the scalar matrix approaches the identity $\mathcal{H}_{A B}(x) \rightarrow \delta_{A B}$, the generalized metric takes the form

$$
\mathcal{H}_{M N}(x, \mathbb{Y}) \rightarrow H_{M N}(\mathbb{Y})=U^{A}{ }_{M}(\mathbb{Y}) \delta_{A B} U^{B}{ }_{N}(\mathbb{Y}) .
$$

Sketched in this form, it is natural to identify the twist matrix $U(\mathbb{Y})$ with a vielbein for the metric $H(\mathbb{Y})$. In fact, this metric is invariant under $O(d) \times O(d)$ transformations 
that preserve the identity and act on the twist from the left, and it transforms under $O(d, d)$ acting on the twists from the right. Therefore, the twists $U(\mathbb{Y})$ can be interpreted as generalized internal vielbeins, associated to the internal geometry on which we are compactifying. Any non-trivial scalar matrix $\mathcal{H}_{A B}(x)$ can later be interpreted as scalar fluctuations deforming the background described by $H(\mathbb{Y})$.

It is useful to introduce the 1 -forms

$$
\Gamma^{A}=U^{A}{ }_{M}(\mathbb{Y}) d \mathbb{Y}^{M}
$$

When the double space is untwisted, these 1-forms are simply the differentials $d \mathbb{Y}^{A}$, which are globally well defined and covariantly constant with respect to the standard derivative $d$. The Scherk-Schwarz twisted torus [40] has instead globally well defined 1-forms, $\Gamma^{a}=$ $U^{a}{ }_{\alpha} d y^{\alpha}$, which are covariantly constant with respect to a derivative that includes a constant non-vanishing Levi-Civita connection $\omega^{a}{ }_{b}$. This becomes manifest through the MaurerCartan equation

$$
d \Gamma^{a}+f_{b c}^{a} \Gamma^{b} \wedge \Gamma^{c}=0 .
$$

Interestingly, $f_{b c}^{a}:=-\omega^{a}{ }_{[b c]}$ can be defined through the Lie bracket that in turn defines the gauge symmetry of the effective action, and in fact, the connection is precisely given by the fluxes. The Scherk-Schwarz twisted torus can be conceived as the manifold of a group, the components of $f$ being its structure constants. Such group is the gauge group of the effective action, where the parameters $f$ appear as gaugings.

We have seen already that many of these features are preserved in our scenario. For instance, the standard Lie bracket is replaced by the C-bracket which determines the gauge invariance of the effective action. However, it is not a priori clear what is the relation between the fluxes $F_{A B C}$ and the connection and torsion of the double twisted torus.

The proper formalism to deal with in the context of double field theory was developed in [38], generalizing the frame-like geometrical formalism introduced by Siegel [47, 48]. It is useful to recall the following definition of the 2-form torsion

$$
T^{a}(V, W)=\nabla_{V} W^{a}-\nabla_{W} V^{a}-[V, W]^{a},
$$

where $V, W$ are two vector fields, $\nabla$ denotes the covariant derivative and [,] the Lie bracket. This can alternatively be written in the more convenient form

$$
T^{a}(V, W)=\left[\mathcal{L}_{V}^{\nabla} W-\mathcal{L}_{V} W\right]^{a},
$$

where $\mathcal{L}_{V}$ is the Lie derivative of vector fields and the upper index $\nabla$ means one must change the partial derivative by the covariant one.

So, we define the generalized torsion in the context of double field theory as in (5.5), where the label $a$ is replaced by $A$ and the standard Lie bracket is replaced by the Cbracket [38] (see [49] for a discussion of torsion in the context of Generalized Geometry). Using the Lie derivative (2.9) and the covariant derivative

$$
\nabla_{M} V^{A}=\partial_{M} V^{A}+\omega^{A}{ }_{B M} V^{B},
$$


where $\omega^{A}{ }_{B}$ represents the generalized spin connection, and imposing the metricity condition (which implies $\omega_{A B}=-\omega_{B A}$ ) one gets

$$
T_{A B C}=\omega_{A C B}+\omega_{B A C}+\omega_{C B A}-\left(\mathcal{L}_{\mathcal{E}_{B}} \mathcal{E}_{C}\right)_{A}=-3 \omega_{[A B C]}+F_{A B C} .
$$

Different connections define different torsions. In the torsionless case, the connection and the fluxes are related through

$$
F_{A B C}=3 \omega_{[A B C]} .
$$

Notice that the antisymmetry of $F_{A B C}$ in all the indices implies

$$
F_{A B}^{A}=0,
$$

which generalizes the equation $f^{\alpha}{ }_{\alpha \beta}=0$, ensuring the invariance of the measure under the killing isometries of geometric backgrounds with $f$ fluxes.

\subsection{Generalized fluxes and superstring compactifications}

The low-energy effective theories arising in standard compactifications of string theory on manifolds with reduced structure or orbifolds are gauged supergravities in which only a subset of gaugings can be obtained. Those gaugings whose origin from a 10-dimensional supergravity has not been identified are dubbed non-geometric. From a stringy perspective, the main motivation for introducing these fluxes is duality. Interestingly, being invariant under T-duality transformations, DFT provides a higher dimensional framework in which all fluxes related to the antisymmetric field strength $H$-flux through T-duality appear on an equal footing.

We have shown in section 4 that the compactified theory can be identified with the bosonic electric sector of $\mathcal{N}=4$ gauged supergravity with broken global symmetry group $G=S L(2, \mathbb{R}) \times O(6,6+N)$. From a stringy perspective, the $\mathcal{N}=4$ supergravity theory could arise, for instance, from the compactification of $D=10$ heterotic string [41], but also from compactifications of Type II either on toroidal orientifolds [7, 50] or on $S U(2)$ structure manifolds [51]. The global group $G$ has different interpretations in each case (see [8] for details).

In the heterotic case, $S L(2, \mathbb{R})$ corresponds to fractional linear transformations of the heterotic axio-dilaton field $\tau_{H}=\lambda+i e^{-2 \phi}$ (where $\lambda$ is the dualized 2-form Kalb-Ramond field) whereas $O(6,6)$ (let us set $N=0$ for the moment) is the group that contains T-duality transformations, as introduced in the context of Generalized Geometry.

However, if we consider Type IIB compactifications, $S L(2, \mathbb{R})$ is the S-duality group that, in particular, acts on the IIB axio-dilaton field $\tau_{B}=C_{0}+i e^{-2 \phi}$ (where $C_{0}$ is the RR 0 -form) as modular transformations. T-duality transformations are more involved here and imply the action of elements of both subgroups in $G_{B}=S L(2, \mathbb{R}) \times O(6,6)_{B}$.

The completion of T-duality transformations to the full $O(6,6)$ group was part of the original motivation for writing down the DFT action (2.4) and, therefore, the identification with a heterotic compactification is naturally suggested. In particular, if the 2-form field $\hat{B}_{\hat{\rho} \hat{\nu}}$ in (3.6) is the Kalb-Ramond field of the heterotic string, we see from (4.17) that 
$\tau=\lambda+i e^{-2 \phi}$ would indeed be the heterotic axio-dilaton field. In what follows we will pursue this interpretation, but we will comment at the end of this section that a Type IIB orientifold interpretation is seemingly possible.

We now address the issue of the $2 d$-dimensional origin of the fluxes. Starting from their definition (3.20):

$$
F_{A B C}=3 \mathcal{I}_{D[A}\left(U^{-1}\right)^{N}{ }_{B}\left(U^{-1}\right)^{P}{ }_{C} \partial_{N} U^{D}{ }_{P},
$$

we parameterize the internal $2 d$-bein in the most general form (see appendix A)

$$
U=\left(\begin{array}{cc}
\left(e^{-1}\right)^{T}(1+B \beta) & -\left(e^{-1}\right)^{T} B \\
-e \beta & e
\end{array}\right), \quad U^{-1}=\left(\begin{array}{cc}
e^{T} & B e^{-1} \\
\beta e^{T} & (1+\beta B) e^{-1}
\end{array}\right) .
$$

One can then define the relation between the tensor $F_{A B C}$ and the standard set of NS-NS string fluxes of [5]. Defining for simplicity $\tilde{a} \equiv a+d$, we set

$$
F_{\tilde{a} \tilde{b} \tilde{c}}=H_{a b c}, \quad F_{\tilde{b} \tilde{c}}^{\tilde{a}}=\omega_{b c}^{a}, \quad F_{\tilde{a}}^{\tilde{a} \tilde{b}}=Q_{c}^{a b}, \quad F^{\tilde{a} \tilde{b} \tilde{c}}=R^{a b c} .
$$

The different fluxes take the following form in terms of this parameterization

$$
\begin{aligned}
& H_{a b c}=3\left\{\partial^{\alpha}\left[(1+\beta B) e^{-1}\right]_{[a}^{\gamma}\left(e^{-T} B\right)_{b \underline{\alpha}}\left(e^{-T} B\right)_{c] \gamma}-\partial^{\alpha}\left(B e^{-1}\right)_{\gamma[a}\left(e^{-T} B\right)_{b \underline{\alpha}}\left[e^{-T}(1+B \beta)\right]_{c]}^{\gamma}\right. \\
& -\partial_{\alpha}\left[(1+\beta B) e^{-1}\right]_{[a}^{\gamma}\left[e^{-T}(1+B \beta)\right]_{b}^{\alpha}\left(e^{-T} B\right)_{c] \gamma} \\
& \left.+\partial_{\alpha}\left(B e^{-1}\right)_{\gamma[a}\left[e^{-T}(1+B \beta)\right]_{b}^{\alpha}\left[e^{-T}(1+B \beta)\right]_{c]}^{\gamma}\right\}, \\
& \omega_{b c}^{a}=-\partial^{\alpha}\left(\beta e^{T}\right)^{\gamma a}\left[e^{-T}(1+B \beta)\right]_{[b}{ }^{\alpha}\left(e^{-T} B\right)_{c] \gamma}-\partial^{\alpha}\left(e^{T}\right)_{\gamma}{ }^{a}\left(e^{-T} B\right)_{[b \underline{\alpha}}\left[e^{-T}(1+B \beta)\right]_{c]}{ }^{\gamma} \\
& -\partial_{\alpha}\left(\beta e^{T}\right)^{\gamma a}\left[e^{-T}(1+B \beta)\right]_{[b}{ }^{\alpha}\left(e^{-T} B\right)_{c] \gamma}+\partial_{\alpha}\left(e^{T}\right)_{\gamma}{ }^{a}\left[e^{-T}(1+B \beta)\right]_{[b}{ }^{\alpha}\left[e^{-T}(1+B \beta)\right]_{c]}{ }^{\gamma} \\
& +e_{\alpha}^{a}\left\{\partial^{[\alpha}\left[(1+\beta B) e^{-1}\right]_{[b}^{\gamma]}\left(e^{-T} B\right)_{c] \gamma}\right. \\
& \left.+\partial_{\gamma}\left[(1+\beta B) e^{-1}\right]_{[b}^{\alpha}\left[e^{-T}(1+B \beta)\right]_{c]}^{\gamma}-\partial^{\alpha}\left(B e^{-1}\right)_{\gamma[b}\left[e^{-T}(1+B \beta)_{c]}^{\gamma}\right]\right\} \\
& -(e \beta)^{a \alpha}\left\{\partial_{[\gamma}\left(B e^{-1}\right)_{\alpha][b}\left[e^{-T}(1+B \beta)\right]_{c]}\right]^{\gamma} \\
& \left.+\partial_{\alpha}\left[(1+\beta B) e^{-1}\right]_{[b}^{\gamma}\left(e^{-T} B\right)_{c] \gamma}-\partial^{\gamma}\left(B e^{-1}\right)_{\alpha[b}\left(e^{-T} B\right)_{c] \gamma}\right\}, \\
& Q_{a}^{b c}=\partial^{\alpha}\left[(1+\beta B) e^{-1}\right]_{a}^{\gamma} e^{[b}{ }_{\alpha} e^{c]}{ }_{\gamma}-\partial^{\alpha}\left(B e^{-1}\right)_{\gamma a} e^{[b}{ }_{\alpha}(e \beta)^{c] \gamma} \\
& -\partial_{\alpha}\left[(1+\beta B) e^{-1}\right]_{a}^{\gamma}(e \beta)^{[b \underline{\alpha}} e_{\gamma}^{c]}+\partial_{\alpha}\left(B e^{-1}\right)_{\gamma a}(e \beta)^{[b \underline{\alpha}}(e \beta)^{c] \gamma} \\
& -\left(e^{-T} B\right)_{a \alpha}\left\{\partial^{[\alpha}\left(\beta e^{T}\right)^{\gamma][c} e_{\gamma}^{b]}+\partial_{\gamma}\left(\beta e^{T}\right)^{\alpha[c}(e \beta)^{b] \gamma}-\partial^{\alpha}\left(e^{T}\right)_{\gamma}^{[c}(e \beta)^{b] \gamma}\right\} \\
& +\left[e^{-T}(1+B \beta)\right]_{a}^{\alpha}\left\{\partial_{[\gamma}\left(e^{T}\right)_{\alpha]}^{[c}(e \beta)^{b] \gamma}+\partial_{\alpha}\left(\beta e^{T}\right)^{\gamma[c} e^{b]}{ }_{\gamma}-\partial^{\gamma}\left(e^{T}\right)_{\alpha}^{[c} e^{b]} \gamma,\right. \\
& R^{a b c}=3 e_{\alpha}^{a} e_{\lambda}^{b} e_{\gamma}^{c}\left\{-\partial_{\delta} \beta^{[\alpha \lambda} \beta^{\gamma] \delta}+\partial^{[\alpha} \beta^{\lambda \gamma]}\right\},
\end{aligned}
$$

where underlined indices must not be antisymmetrized.

It is interesting to analyze the dependence of the fluxes in different limits of interest:

- Setting $\beta=0$ and taking $B$, $e$ to depend only on base coordinates, the $H$-flux takes the geometric form

$$
H_{a b c}=3\left(e^{-1}\right)^{\alpha}{ }_{a}\left(e^{-1}\right)^{\beta}{ }_{b}\left(e^{-1}\right)^{\gamma}{ }_{c} \partial_{[\alpha} B_{\beta \gamma]} .
$$


Gauge transformations for $B \rightarrow B+d \Lambda$ are given by $O(d, d) B$-type transformations acting from the right

$$
\begin{aligned}
\left(\begin{array}{cc}
\left(e^{-1}\right)^{T}-\left(e^{-1}\right)^{T} B \\
0 & e
\end{array}\right) \rightarrow & \left(\begin{array}{cc}
\left(e^{-1}\right)^{T} & -\left(e^{-1}\right)^{T} B \\
0 & e
\end{array}\right)\left(\begin{array}{cc}
1 & -d \Lambda \\
0 & 1
\end{array}\right) \\
& =\left(\begin{array}{cc}
\left(e^{-1}\right)^{T} & -e^{-1}(B+d \Lambda) \\
0 & e
\end{array}\right)
\end{aligned}
$$

and it can be seen from (5.17) that they leave the gaugings invariant.

- Setting $B=\beta=0$ and taking $e$ to depend only on base coordinates, the $\omega$-flux takes the simple form

$$
\omega_{b c}^{a}=2\left(e^{-1}\right)_{[b}^{\alpha}\left(e^{-1}\right)^{\gamma}{ }_{c]} \partial_{\alpha} e_{\gamma}^{a} .
$$

Or also, by letting $B$ depend on dual coordinates and setting $e=\mathbf{1}$ and $\beta=0$, one gets

$$
\omega_{b c}^{a}=\partial^{a} B_{b c} .
$$

- Setting $e=1, B=0$ and $\beta$ depending only on base coordinates, the $Q$-flux reads

$$
Q_{c}^{a b}=\partial_{c} \beta^{a b}
$$

Also setting $\beta=B=0$ and making $e$ depend on dual coordinates only, one gets

$$
Q_{c}^{a b}=2 e^{[a}{ }_{\alpha} e^{b]}{ }_{\gamma} \partial^{\alpha}\left(e^{-1}\right)^{\gamma}{ }_{c} .
$$

Notice that, from the first and third terms in (5.15) and keeping only ordinary derivatives, we obtain $Q_{c}^{a b}=\beta^{r a} \beta^{b s} H_{r s c}+\ldots$ reproducing the expression found in [11] (see also [24]).

- The $R$-flux can be obtained by setting $e=\mathbf{1}, B=0$ and taking $\beta$ to depend only on dual coordinates, so that

$$
R^{a b c}=3 \partial^{[a} \beta^{b c]} .
$$

An interesting observation from eq. (5.16) is that any configuration defined by $\beta(y)$ leads to a non-vanishing R-flux. Since locally $\beta$-transformations can be gauged away, one could hope to construct locally geometric backgrounds associated to the R-flux.

The $O(d, d)$ covariance is responsible for the symmetry among (5.17), (5.18), (5.19) and $(5.20),(5.21),(5.22)$, respectively.

Including extra vector fields as in subsection 4.2 .2 , one can explicitly write the fluxes carrying indices $M=2 d+N$. It is interesting to see that setting $B=0, \beta=0$ one recovers the electromagnetic fluxes. Indeed, one finds

$$
\mathcal{F}_{a b}^{i} \equiv F_{(2 d+i) \tilde{a} \tilde{b}}=2\left(e^{-1}\right)^{\alpha}{ }_{a}\left(e^{-1}\right)^{\beta}{ }_{b} \partial_{[\alpha} V_{\beta]}^{i} .
$$


Having made contact with string fluxes, we now define the subgroup of $O(d, d)$ transformations whose elements correspond to T-dualities, namely

$$
\left(T_{\alpha}\right)^{N}{ }_{M}=\delta^{N}{ }_{M}-\delta^{N, \alpha} \delta_{M, \alpha}-\delta^{N, \alpha+d} \delta_{M, \alpha+d}+\delta^{N, \alpha+d} \delta_{M, \alpha}+\delta^{N, \alpha} \delta_{M, \alpha+d}
$$

where the index $\alpha$ denotes the direction in which the T-duality is performed. As expected, these elements satisfy the following properties

$$
\left[T_{\alpha}, T_{\beta}\right]=0, \quad\left(T_{\alpha}\right)^{N}{ }_{P}\left(T_{\alpha}\right)^{P}{ }_{M}=\delta^{N}{ }_{M}
$$

The T-duality operators (5.24) lead to the Buscher rules introduced in [52]. We have seen in (2.7) how the generalized metric transforms under these symmetries. Equivalently, one can define a unified tensor $E=g+B$ [53], which transforms as

$$
U=\left(\begin{array}{ll}
a & b \\
c & d
\end{array}\right), \quad E^{\prime}=(a E+b)(c E+d)^{-1}
$$

Taking the $O(d, d)$ transformation to be a T-duality defined in (5.24) in the $y^{\alpha}$ direction, one obtains the following transformations for $E$

$$
E_{\alpha \alpha}^{\prime}=\frac{1}{E_{\alpha \alpha}}, \quad E_{\beta \alpha}^{\prime}=\frac{E_{\beta \alpha}}{E_{\alpha \alpha}}, \quad E_{\alpha \beta}^{\prime}=-\frac{E_{\alpha \beta}}{E_{\alpha \alpha}}, \quad E_{\beta \gamma}^{\prime}=E_{\beta \gamma}-\frac{E_{\beta \alpha} E_{\alpha \gamma}}{E_{\alpha \alpha}},
$$

which decomposed into symmetric and anti-symmetric parts lead to the well-known Buscher rules

$$
\begin{array}{rlrl}
g_{\alpha \alpha}^{\prime} & =\frac{1}{g_{\alpha \alpha}}, & g_{\alpha \beta}^{\prime}=-\frac{B_{\alpha \beta}}{g_{\alpha \alpha}}, & g_{\beta \gamma}^{\prime}=g_{\beta \gamma}-\frac{g_{\alpha \beta} g_{\alpha \gamma}-B_{\alpha \beta} B_{\alpha \gamma}}{g_{\alpha \alpha}} \\
B_{\alpha \beta}^{\prime} & =-\frac{g_{\alpha \beta}}{g_{\alpha \alpha}}, & B_{\beta \gamma}^{\prime}=B_{\beta \gamma}-\frac{g_{\alpha \beta} B_{\alpha \gamma}-B_{\alpha \beta} g_{\alpha \gamma}}{g_{\alpha \alpha}} .
\end{array}
$$

Defined like this, the standard T-duality chain [5] holds

$$
H_{a b c} \stackrel{T_{a}}{\longleftrightarrow} \omega_{b c}^{a} \stackrel{T_{b}}{\longleftrightarrow} Q_{c}^{a b} \stackrel{T_{c}}{\longleftrightarrow} R^{a b c},
$$

and when the Jacobi identities (3.35) are written in terms of the fluxes (5.13), the following results of [5] are exactly recovered

$$
\begin{aligned}
H_{e[a b} \omega_{c d]}^{e} & =0, \\
\omega_{b[c}^{a} \omega_{d e]}^{b}+H_{b[c d} Q_{e]}^{a b} & =0, \\
Q_{c}^{[a b]} \omega_{[d e]}^{c}-4 \omega_{c[d}^{[a} Q_{e]}^{b] c}+H_{c[d e]} R^{[a b] c} & =0, \\
Q_{c}^{[a b} Q_{e}^{d] c}+\omega_{c e}^{[a} R^{b d] c} & =0, \\
Q_{c}^{[a b} R^{d e] c} & =0 .
\end{aligned}
$$


This set of equations closes under T-duality transformations (5.24), as expected from the fact that (3.35) is $O(d, d)$ covariant. Note that defining $\mathcal{E}_{a}=-Z_{a}$ and $\mathcal{E}^{a}=-X^{a}$ the T-covariant algebra of [5] is also recovered

$$
\begin{aligned}
{\left[Z_{a}, Z_{b}\right] } & =H_{a b c} X^{c}+\omega_{a b}^{c} Z_{c}, \\
{\left[Z_{a}, X^{b}\right] } & =-\omega_{a c}^{b} X^{c}+Q_{a}^{b c} Z_{c}, \\
{\left[X^{a}, X^{b}\right] } & =Q_{c}^{a b} X^{c}+R^{a b c} Z_{c} .
\end{aligned}
$$

Interestingly enough, also the extra gauge vectors fit in this description. This can be seen by using the Jacobi identity (3.35) with generic fluxes including indices $i=1 \ldots N$ for vectors. Indeed, by keeping (for simplicity) only $\omega_{a b}^{c}, H_{a b c}$ and $\mathcal{F}_{a b}^{i}$ fluxes we obtain

$$
\begin{aligned}
\omega_{[a b}^{d} \omega_{c] d}^{e} & =0, \\
\omega_{[a b}^{d} \mathcal{F}_{c] d}^{i} & =0, \\
H_{e[a b} \omega_{c d]}^{e} & =-\mathcal{F}_{[a b}^{i} \mathcal{F}_{c d]}^{i},
\end{aligned}
$$

which are the Jacobi identities derived from the heterotic algebra found in [41], after adjusting some normalization factors.

\subsection{Type I interpretation}

As we mentioned at the beginning of this section, the effective DFT four dimensional theory can be equally well interpreted in terms of a Type IIB orientifold compactification. For instance, if instead of the identifications (5.13) we had used

$$
F_{\tilde{a} \tilde{b} \tilde{c}}=\mathbf{F}_{a b c}, \quad F_{\tilde{b} \tilde{c}}^{\tilde{a}}=\omega_{b c}^{a}, \quad F_{\tilde{a} \tilde{b}}^{(2 d+i)}=\mathcal{F}_{a b}^{i},
$$

where now $\mathbf{F}_{a b c}$ is the RR 3-form flux of Type IIB compactification with O9 planes, $\omega_{b c}^{a}$ is a geometric flux and for simplicity we turn off the non-geometric fluxes. In this case, the Jacobi identities would read

$$
\begin{aligned}
\omega_{[a b}^{d} \omega_{c] d}^{e} & =0 \\
\omega_{[a b}^{d} \mathcal{F}_{c] d}^{i} & =0 \\
\mathbf{F}_{e[a b} \omega_{c d]}^{e} & =-\mathcal{F}_{[a b}^{i} \mathcal{F}_{c d]}^{i},
\end{aligned}
$$

where the first equation is the usual constraint on geometric fluxes, the second equation is the requirement of absence of Freed-Witten anomalies and the third one is a tadpole cancelation equation [7] if vector fluxes are now interpreted as D9 magnetic fluxes.

The full set of Type IIB generalized fluxes can be identified (see for instance [7] in an O3 notation). For bulk fluxes, they correspond to a definite $S L(2, \mathbb{R})$ spin (say plus in the notation of section 4$)$ in the $(\mathbf{2}, \mathbf{2 2 0})$ representation of $S L(2, \mathbb{R}) \times O(6,6)_{B}$. The opposite spin would correspond to S-dual fluxes.

From the point of view of the starting DFT, the original fields have now a different interpretation. Namely, the $\hat{B}_{\hat{\rho} \hat{\nu}}$ field entering the generalized metric $\hat{\mathcal{H}}_{\hat{M} \hat{N}}$ in (2.1) is such 
that in the ten dimensional effective field theory it reproduces the two form RR field. Namely, the DFT effective theory in ten space-time dimensions now reads

$$
S_{10}=\int d^{10} x \sqrt{|g(x)|} e^{-2 \Phi(x)}\left\{\mathbf{R}+4 \partial^{\mu} \Phi \partial_{\mu} \Phi-\frac{1}{12} F^{2}\right\},
$$

where $F$ is the RR 3-form field strength.

\section{Conclusions}

We have performed a generalized Scherk-Schwarz compactification of DFT and obtained the dimensionally reduced effective action in arbitrary number of dimensions. The reduced action has $O(d, d)$ symmetry broken by the fluxes and a gauge symmetry, both inherited from the global $O(D, D)$ and gauge invariance of DFT, respectively. The structure constants of the gauge group are defined by the C-bracket and we have given their explicit definition in terms of the twists. We have shown that, although the C-bracket does not generically satisfy the Jacobi identity, it leads to gaugings which verify such identity when particularly evaluated in terms of the twists.

Specified to four space-time and six internal dimensions, the effective action reproduces the bosonic electric sector of gauged $\mathcal{N}=4$ supergravity. The comparison was made with the formulation of [42], and the precise dualizations required to make the equivalence explicit can be found in section 4 . Furthermore, the global symmetry group of the effective action was enhanced to $O(d, d+N)$, by considering, as a starting point, the abelian heterotic extension of DFT formulated in [30]. In addition, the gauging of a generalization of the rescaling symmetry considered in [46] was proposed as an origin for the $\xi_{+}^{M}$ fluxes. Altogether, our results imply that all the electric gaugings of $\mathcal{N}=4$ gauged supergravity can be reached from DFT.

It is worth mentioning that throughout the paper a strong constraint, as discussed in section 2, was assumed. This is needed for gauge invariance of the DFT original action. Interestingly enough the Scherk-Schwarz like dimensional reduction to go from DFT action (2.4) to the effective action (3.25) does not require further use of the strong constraint. ${ }^{3}$ This also applies, in particular, for Jacobi identities implying the " $\mathcal{N}=4$ quadratic constraints", needed in gauged supergravities for the closure of the gauge transformations. A similar observation was pointed out in [55] (see also [29]). It would then be interesting to analyze in the future if this is an indication of some possible consistent relaxation of the strong constraint of the starting DFT.

The generalized Scherk-Schwarz reduction can be interpreted as a compactification on a twisted double torus, its vielbein being defined by the twist matrix. A precise definition of torsion in terms of the connection allows to relate these geometric concepts with the fluxes. Moreover, the standard NS-NS (non)-geometric fluxes $(H, \omega, Q, R)$ can be identified with the gaugings of the effective action, and the string $d$-dimensional background can be decoded from the double twisted $2 d$-torus. The fluxes obey the standard $T$-duality

\footnotetext{
${ }^{3}$ In the $\xi_{+M} \neq 0$ case, level matching is not enough and extra requirements, but still weaker than the strong constraint, are needed.
} 
chain and satisfy Jacobi identities reproducing the results of [5]. In this way, the higher dimensional origin of the string fluxes can be traced to the new degrees of freedom of DFT.

Finally, we have presented a novel interpretation of DFT in terms of Type IIB/O9 orientifolds. Identifying the Kalb-Ramond $B$-field with the RR 2-form $C_{2}$, the 10-dimensional action of the Type I string can be obtained from DFT when dual coordinates are turned off. This requires a reinterpretation of the $O(D, D)$ symmetry, which is no longer trivially associated with (the heterotic) T-duality. Also in this case the gaugings can be identified with fluxes, and some of the quadratic constraints can be interpreted in terms of tadpole cancelation conditions and Freed-Witten anomalies.

\section{Acknowledgments}

We are very grateful to M. Graña for valuable comments on the manuscript. We also thank A. Rosabal and H. Triendl for useful discussions and comments. G. A., W.B. and C.N. thank IPhT- Saclay, where part of this work was done, for hospitality. This work was supported by CONICET PIP 112-200801-00507, MINCYT (Ministerio de Ciencia, Tecnología e Innovación Productiva of Argentina), ECOS-Sud France binational collaboration project A08E06, University of Buenos Aires UBACyT X161 and the ERC Starting Independent Researcher Grant 259133 - ObservableString.

\section{A Gauging away $\beta$-transformations}

In this appendix we closely follow the results of [11]. The aim is to make the structure of the vielbein explicit. The internal vielbein $\mathcal{E}^{A}{ }_{M}(x, \mathbb{Y})$ which contains the fluctuations around $U^{A}{ }_{M}(\mathbb{Y})$ (the vielbein of the background) is an element of $G / K$, namely its planar index rotates under gauge transformations $K=O(d) \times O(d)$ from the left, and its curved index rotates under global $G=O(d, d)$ transformations from the right. Any two configurations that are connected by elements of $K$ physically correspond to the same configuration. Given that $\operatorname{dim}(G)=(2 d-1) d$ and $\operatorname{dim}(K)=d(d-1) / 2+d(d-1) / 2$, then $\operatorname{dim}(G / K)=d^{2}$, of which $d(d+1) / 2$ degrees of freedom are parameterized by a metric field $h(x, \mathbb{Y})$, and the remaining $d(d-1) / 2$ are parameterized by an antisymmetric $b(x, \mathbb{Y})$-field. A possible and convenient general parameterization of $\tilde{\mathcal{E}}$ is given by

$$
\tilde{\mathcal{E}}=\frac{1}{\sqrt{2}}\left(\begin{array}{c}
e_{+}-e_{+}(b+h) \\
e_{-}-e_{-}(b-h)
\end{array}\right)
$$

which, restricted to $h^{-1}=e_{ \pm}^{T} e_{ \pm}$, leads to the usual form of the generalized metric

$$
\mathcal{H}(x, \mathbb{Y})=\tilde{\mathcal{E}}^{T} \tilde{\mathcal{E}}=\left(\begin{array}{cc}
h^{-1} & -h^{-1} b \\
b h^{-1} & h-b h^{-1} b
\end{array}\right) .
$$

Given any element $\tilde{\mathcal{O}} \in K$ of the form

$$
\tilde{\mathcal{O}}=\left(\begin{array}{cc}
O_{+} & 0 \\
0 & O_{-}
\end{array}\right),
$$


acting on $\tilde{\mathcal{E}}$ from the left leaves the generalized metric invariant, but rotates $e_{ \pm}$. Notice that the vielbein (A.1) is such that

$$
\mathcal{I}=\tilde{\mathcal{E}}^{T}\left(\begin{array}{cc}
-1 & 0 \\
0 & 1
\end{array}\right) \tilde{\mathcal{E}}
$$

so (A.1) is clearly not an element of $O(d, d)$. However, it has the advantage that one knows how $O(d) \times O(d)$ transformations act on it. To take it to an $O(d, d)$ form preserving the off-diagonal metric that we use in this paper, $\tilde{\mathcal{E}}$ should be rotated by an $S O(2 d)$ transformation $R$,

$$
R=\frac{1}{\sqrt{2}}\left(\begin{array}{cc}
1 & 1 \\
-1 & 1
\end{array}\right), \quad \mathcal{E}=R \tilde{\mathcal{E}}, \quad \mathcal{I}=\mathcal{E}^{T} \mathcal{I} \mathcal{E},
$$

and this allows to determine the form of a generic $O(d) \times O(d)$ rotation in our case

$$
\mathcal{O}=R \tilde{\mathcal{O}} R^{T}=\frac{1}{2}\left(\begin{array}{c}
O_{+}+O_{-} O_{-}-O_{+} \\
O_{-}-O_{+} O_{+}+O_{-}
\end{array}\right) .
$$

Note also that the form of the vielbein is now

$$
\mathcal{E}=\frac{1}{2}\left(\begin{array}{cc}
e_{+}+e_{-} & -\left(e_{+}+e_{-}\right) b+\left(e_{-}-e_{+}\right) h \\
e_{-}-e_{+} & \left(e_{+}-e_{-}\right) b+\left(e_{+}+e_{-}\right) h
\end{array}\right),
$$

and we can perform a $K$ transformation with $O_{ \pm}$acting on $e_{ \pm}$to set $e_{+}=e_{-}=\left(e^{-1}\right)^{T}$, and take $\mathcal{E}$ to triangular form

$$
\left(\begin{array}{cc}
\left(e^{-1}\right)^{T} & -\left(e^{-1}\right)^{T} b \\
0 & e
\end{array}\right)=\left(\begin{array}{cc}
\left(e^{-1}\right)^{T} & 0 \\
0 & e
\end{array}\right)\left(\begin{array}{cc}
1 & -b \\
0 & 1
\end{array}\right) .
$$

We have then managed to write it as a product of diffeomorphisms and B-transformations. Any $\beta$-transformation

$$
\mathcal{E}_{\beta}=\left(\begin{array}{cc}
1 & 0 \\
-\beta & 1
\end{array}\right)
$$

would take it away from the triangular gauge, to a form parameterizable as in (A.7), so it can always be brought to triangular form through a $K$-rotation. It is worth emphasizing that, although the $K$-transformation seems to restore the effect of the $\beta$-transformation, the restoration is only structural, and in many cases it is performed at the expense of ending with non-geometric backgrounds $h$ and $b$. In this paper we have chosen the triangular gauge to do the computations, but the results are finally written in a covariant way.

Note that the gaugings are not invariant under $O(d) \times O(d)$ transformations of the vielbein, so even if it might seem that two physically equivalent vielbeins give rise to different theories, the gaugings should be computed through different expressions. 


\section{B A single flux example}

Here we would like to consider simple backgrounds, on which we apply T-dualities and track how the obtained (non)geometries are encoded in the double torus. For simplicity we turn on a unique flux for which all the consistency equations are automatically satisfied. We closely follow the procedure of [54], where it is analyzed how the different fluxes twist the double torus through successive T-dualizations and how geometric and non-geometric backgrounds are obtained after projecting the different twisted double tori to a base. Although the definition of flux is different here than in [54], we show that the conclusions remain unaltered.

\section{B.1 H-flux}

The starting point is a double three-torus $T^{3} \times \tilde{T}^{3}=\left(y^{1}, y^{2}, y^{3}, y^{4}, y^{5}, y^{6}\right)$ with H-flux over $T^{3}$ in Type IIB. Although the base is 3-dimensional, we are assuming that this space can be fibered over some other space to render a proper 6-dimensional string background. We begin with a simple $T^{3}$ with all radii equal to unity, described by $U=\mathbf{1}_{6}$, and perform a $B$-transformation to introduce $H_{123}=3 N$ units of H-flux in $T^{3}$, namely

$$
d s^{2}=\left(d y^{1}\right)^{2}+\left(d y^{2}\right)^{2}+\left(d y^{3}\right)^{2}, \quad B=6 N y^{[3} d y^{1} \wedge d y^{2]} .
$$

This leads to the twist matrix

$$
U_{H}=\left(\begin{array}{cccccc}
1 & 0 & 0 & 0 & -N y^{3} & N y^{2} \\
0 & 1 & 0 & N y^{3} & 0 & -N y^{1} \\
0 & 0 & 1 & -N y^{2} & N y^{1} & 0 \\
0 & 0 & 0 & 1 & 0 & 0 \\
0 & 0 & 0 & 0 & 1 & 0 \\
0 & 0 & 0 & 0 & 0 & 1
\end{array}\right)
$$

that allows to define a generalized coframe $\Gamma^{A}=U^{A}{ }_{P} d \mathbb{Y}^{P}$

$$
\begin{aligned}
& \Gamma^{1}=d y^{1}, \quad \Gamma^{2}=d y^{2}, \quad \Gamma^{3}=d y^{3}, \\
& \Gamma^{4}=d y^{4}-N y^{3} d y^{2}+N y^{2} d y^{3} \\
& \Gamma^{5}=d y^{5}+N y^{3} d y^{1}-N y^{1} d y^{3} \\
& \Gamma^{6}=d y^{6}-N y^{2} d y^{1}+N y^{1} d y^{2}
\end{aligned}
$$

The identifications that make the one-forms (B.3) globally well defined are given by

$$
\begin{array}{ll}
\left(y^{1}, y^{5}, y^{6}\right) \rightarrow\left(y^{1}+1, y^{5}+N y^{3}, y^{6}-N y^{2}\right), & y^{4} \rightarrow y^{4}+1, \\
\left(y^{2}, y^{4}, y^{6}\right) \rightarrow\left(y^{2}+1, y^{4}-N y^{3}, y^{6}+N y^{1}\right), & y^{5} \rightarrow y^{5}+1, \\
\left(y^{3}, y^{4}, y^{5}\right) \rightarrow\left(y^{3}+1, y^{4}+N y^{2}, y^{5}-N y^{1}\right), & y^{6} \rightarrow y^{6}+1 .
\end{array}
$$

One can define three kahler moduli as

$$
\rho_{i}=\frac{1}{2} \epsilon_{i j k} B_{j k}+i \operatorname{Vol}\left(T_{i}^{2}\right)
$$

and check that under the monodromies $y^{i} \rightarrow y^{i}+1$ in (B.4) they shift as $\rho_{i} \rightarrow \rho_{i}+N$. 


\section{B.2 $\omega$-flux}

Now we apply a T-duality in the direction $y^{3}$ leading to a IIA background with flux $\omega_{12}^{3}=$ $3 N$ where

$$
U_{\omega}=\left(\begin{array}{cccccc}
1 & 0 & N y^{2} & 0 & -N y^{6} & 0 \\
0 & 1 & -N y^{1} & N y^{6} & 0 & 0 \\
0 & 0 & 1 & 0 & 0 & 0 \\
0 & 0 & 0 & 1 & 0 & 0 \\
0 & 0 & 0 & 0 & 1 & 0 \\
0 & 0 & 0 & -N y^{2} & N y^{1} & 1
\end{array}\right)
$$

The one forms

$$
\begin{aligned}
& \Gamma^{1}=d y^{1}, \quad \Gamma^{2}=d y^{2}, \quad \Gamma^{6}=d y^{6} \\
& \Gamma^{4}=d y^{4}-N y^{6} d y^{2}+N y^{2} d y^{6} \\
& \Gamma^{5}=d y^{5}+N y^{6} d y^{1}-N y^{1} d y^{6} \\
& \Gamma^{3}=d y^{3}-N y^{2} d y^{1}+N y^{1} d y^{2}
\end{aligned}
$$

are now globally well behaved under the shifts

$$
\begin{array}{ll}
\left(y^{1}, y^{5}, y^{3}\right) \rightarrow\left(y^{1}+1, y^{5}+N y^{6}, y^{3}-N y^{2}\right), & y^{4} \rightarrow y^{4}+1 \\
\left(y^{2}, y^{4}, y^{3}\right) \rightarrow\left(y^{2}+1, y^{4}-N y^{6}, y^{3}+N y^{1}\right), & y^{5} \rightarrow y^{5}+1 \\
\left(y^{6}, y^{4}, y^{5}\right) \rightarrow\left(y^{6}+1, y^{4}+N y^{2}, y^{5}-N y^{1}\right), & y^{3} \rightarrow y^{3}+1
\end{array}
$$

One can now read the new background from (B.6). After projecting to the base $y^{6}=0$, it is as expected a so-called twisted torus

$$
d s^{2}=\left(d y^{1}\right)^{2}+\left(d y^{2}\right)^{2}+\left(d y^{3}-N y^{2} d y^{1}+N y^{1} d y^{2}\right)^{2}, \quad B=0 .
$$

This can also be obtained by applying a T-duality (5.28) to (B.1). Note that when inserting (B.9) into (5.18), one obtains $\omega_{12}^{3}=2 N$. The fact that the flux is reduced by a unit of $N$ is a reflection of the projection to the base.

Acting with T-duality over (B.5), the IIB kahler moduli $\rho_{i}$ are maped into the complex structure moduli $\tau_{i}$ of the twisted torus in IIA. In particular, the real part $\operatorname{Re}\left(\tau_{1}\right)=-y_{3} / y_{2}$ shifts as $\tau_{1} \rightarrow \tau_{1}+N$ when $y^{1} \rightarrow y^{1}+1$. Also, under the shift $y^{1} \rightarrow y^{1}+1$, the $\mathcal{S} \in O(2,2)$ transformation needed to bring the metric to its original form is block diagonal and does not mix the metric with the $B$-field, i.e.

$$
\mathcal{S}=\left(\begin{array}{cc}
S^{-T} & 0 \\
0 & S
\end{array}\right), \quad S=\left(\begin{array}{ccc}
1 & 0 & 0 \\
0 & 1 & 0 \\
0 & -N & 1
\end{array}\right), \quad U_{\omega}\left(y^{1}+1\right) \mathcal{S}=U_{\omega}\left(y^{1}\right)
$$




\section{B.3 $Q$-flux}

Now we apply a T-duality in the direction $y^{2}$ and obtain a background with flux $Q_{1}^{23}=3 N$ where

$$
U_{Q}=\left(\begin{array}{cccccc}
1 & -N y^{6} & N y^{5} & 0 & 0 & 0 \\
0 & 1 & 0 & 0 & 0 & 0 \\
0 & 0 & 1 & 0 & 0 & 0 \\
0 & 0 & 0 & 1 & 0 & 0 \\
0 & 0 & -N y^{1} & N y^{6} & 1 & 0 \\
0 & N y^{1} & 0 & -N y^{5} & 0 & 1
\end{array}\right)
$$

with globally well defined one-forms on the double torus

$$
\begin{aligned}
& \Gamma^{1}=d y^{1}, \quad \Gamma^{5}=d y^{5}, \quad \Gamma^{6}=d y^{6} \\
& \Gamma^{4}=d y^{4}-N y^{6} d y^{5}+N y^{5} d y^{6} \\
& \Gamma^{2}=d y^{2}+N y^{6} d y^{1}-N y^{1} d y^{6} \\
& \Gamma^{3}=d y^{3}-N y^{5} d y^{1}+N y^{1} d y^{5}
\end{aligned}
$$

under the monodromies

$$
\begin{array}{ll}
\left(y^{1}, y^{2}, y^{3}\right) \rightarrow\left(y^{1}+1, y^{2}+N y^{6}, y^{3}-N y^{5}\right), & y^{4} \rightarrow y^{4}+1 \\
\left(y^{5}, y^{4}, y^{3}\right) \rightarrow\left(y^{5}+1, y^{4}-N y^{6}, y^{3}+N y^{1}\right), & y^{2} \rightarrow y^{2}+1 \\
\left(y^{6}, y^{4}, y^{2}\right) \rightarrow\left(y^{6}+1, y^{4}+N y^{5}, y^{2}-N y^{1}\right), & y^{3} \rightarrow y^{3}+1 .
\end{array}
$$

The matrix (B.11) does not have the proper triangular form to read off the background. To take it to such form, we perform an $O(3) \times O(3)$ rotation acting on the left, defined by

$$
\mathcal{O}=\frac{1}{2}\left(\begin{array}{c}
O_{+}+O_{-} O_{-}-O_{+} \\
O_{-}-O_{+} O_{+}+O_{-}
\end{array}\right), \quad O_{\alpha}=\left(\begin{array}{ccc}
1 & 0 & 0 \\
0 & \gamma & -\alpha N y_{1} \gamma \\
0 & \alpha N y_{1} \gamma & \gamma
\end{array}\right)
$$

with $\gamma \equiv 1 / \sqrt{1+\left(N y^{1}\right)^{2}}$, leading to

$$
\mathcal{O} U_{Q}=\left(\begin{array}{cccccc}
1 & -N y^{6} & N y^{5} & 0 & 0 & 0 \\
0 & 1 / \gamma & 0 & -N^{2} y^{1} y^{5} \gamma & 0 & N y^{1} \gamma \\
0 & 0 & 1 / \gamma & -N^{2} y^{1} y^{6} \gamma & -N y^{1} \gamma & 0 \\
0 & 0 & 0 & 1 & 0 & 0 \\
0 & 0 & 0 & N y^{6} \gamma & \gamma & 0 \\
0 & 0 & 0 & -N y^{5} \gamma & 0 & \gamma
\end{array}\right)
$$

We then see that the background is locally described by

$$
d s^{2}=\left(d y^{1}\right)^{2}+\frac{1}{1+\left(N y^{1}\right)^{2}}\left(\left(d y^{2}\right)^{2}+\left(d y^{3}\right)^{2}\right), \quad B=-\frac{N y^{1}}{1+\left(N y^{1}\right)^{2}} d y^{2} \wedge d y^{3},
$$

which can also be obtained by application of Buscher rules. Again, if the fluxes are computed using this projected background, they are reduced by one unit of $N$, i.e. $Q_{3}^{12}=N$. 
Now we can compute the IIB kahler modulus and its shift under $y^{1} \rightarrow y^{1}+1$ as

$$
\rho_{1}=\frac{-1}{N y^{1}+i}, \quad \frac{1}{\rho_{1}} \rightarrow \frac{1}{\rho_{1}}-N,
$$

and this is again a fiber of $T_{1}^{2}$ shifting the kahler modulus as we move in the direction $y^{1}$. Although this background is locally described in terms of a metric and a B-field, upon going around a cycle $y^{1} \rightarrow y^{1}+1$, the metric and B-field mix through an $\mathcal{S} \in O(2,2)$ transformation $\mathcal{O} U_{Q}\left(y^{1}+1\right) \mathcal{S}=\mathcal{O} U_{Q}\left(y^{1}\right)$, defined by

$$
\mathcal{S}=\left(\begin{array}{cccccc}
1 & 0 & 0 & 0 & 0 & 0 \\
0 & \frac{\gamma\left(y^{1}+1\right)}{\gamma\left(y^{1}\right)} & 0 & 0 & 0 & -N \gamma\left(y^{1}\right) \gamma\left(y^{1}+1\right) \\
0 & 0 & \frac{\gamma\left(y^{1}+1\right)}{\gamma\left(y^{1}\right)} & 0 & N \gamma\left(y^{1}+1\right) \gamma\left(y^{1}\right) & 0 \\
0 & 0 & 0 & 1 & 0 & 0 \\
0 & 0 & 0 & 0 & \frac{\gamma\left(y^{1}\right)}{\gamma\left(y^{1}+1\right)} & 0 \\
0 & 0 & 0 & 0 & 0 & \frac{\gamma\left(y^{1}\right)}{\gamma\left(y^{1}+1\right)}
\end{array}\right)
$$

where we have already projected to the base. The fact that under a cycle the metric and $B$-field mix implies that this background is not globally well defined. However, as seen, locally it is described by a metric and a $B$-field. Therefore, these backgrounds are said to be locally geometric, but globally non-geometric.

\section{B.4 R-flux}

Finally, we apply the remaining T-duality in the direction $y^{1}$ to obtain a background with flux $R^{123}=3 N$ where

$$
U_{R}=\left(\begin{array}{cccccc}
1 & 0 & 0 & 0 & 0 & 0 \\
0 & 1 & 0 & 0 & 0 & 0 \\
0 & 0 & 1 & 0 & 0 & 0 \\
0 & -N y^{6} & N y^{5} & 1 & 0 & 0 \\
N y^{6} & 0 & -N y^{4} & 0 & 1 & 0 \\
-N y^{5} & N y^{4} & 0 & 0 & 0 & 1
\end{array}\right)
$$

This defines the one-forms

$$
\begin{aligned}
& \Gamma^{4}=d y^{4}, \quad \Gamma^{5}=d y^{5}, \quad \Gamma^{6}=d y^{6} \\
& \Gamma^{1}=d y^{1}-N y^{6} d y^{5}+N y^{5} d y^{6} \\
& \Gamma^{2}=d y^{2}+N y^{6} d y^{4}-N y^{4} d y^{6} \\
& \Gamma^{3}=d y^{3}-N y^{5} d y^{4}+N y^{4} d y^{5}
\end{aligned}
$$

and the monodromies

$$
\begin{array}{ll}
\left(y^{4}, y^{2}, y^{3}\right) \rightarrow\left(y^{4}+1, y^{2}+N y^{6}, y^{3}-N y^{5}\right), & y^{1} \rightarrow y^{1}+1 \\
\left(y^{5}, y^{1}, y^{3}\right) \rightarrow\left(y^{5}+1, y^{1}-N y^{6}, y^{3}+N y^{4}\right), & y^{2} \rightarrow y^{2}+1 \\
\left(y^{6}, y^{1}, y^{2}\right) \rightarrow\left(y^{6}+1, y^{1}+N y^{5}, y^{2}-N y^{4}\right), & y^{3} \rightarrow y^{3}+1 .
\end{array}
$$


The matrix $U_{R}$ reduces to the identity when projected to the base, and must then be associated to a trivial torus with vanishing $B$-field. This can be understood if we take into account that in every step of the T-duality procedure we project out the new dual coordinates, and this successively reduces the flux by units of $N$. Since we started from a background with $H_{123}=3 N$ and performed three T-dualities, we have ended after three successive projections with a background having $R^{123}=0$. In summary, T-dualities incorporate dual fluxes into the game which we are forced to project out if we want to make contact with some string background geometry. In this example, this can be done for $H, \omega$ and $Q$ but not for $R$ which then receives the name of locally non-geometric flux.

Open Access. This article is distributed under the terms of the Creative Commons Attribution Noncommercial License which permits any noncommercial use, distribution, and reproduction in any medium, provided the original author(s) and source are credited.

\section{References}

[1] M. Graña, Flux compactifications in string theory: a comprehensive review, Phys. Rept. 423 (2006) 91 [hep-th/0509003] [INSPIRE].

[2] M.R. Douglas and S. Kachru, Flux compactification, Rev. Mod. Phys. 79 (2007) 733 [hep-th/0610102] [INSPIRE].

[3] R. Blumenhagen, B. Körs, D. Lüst and S. Stieberger, Four-dimensional string compactifications with D-branes, orientifolds and fluxes, Phys. Rept. 445 (2007) 1 [hep-th/0610327] [INSPIRE].

[4] H. Samtleben, Lectures on gauged supergravity and flux compactifications, Class. Quant. Grav. 25 (2008) 214002 [arXiv: 0808.4076] [INSPIRE].

[5] J. Shelton, W. Taylor and B. Wecht, Nongeometric flux compactifications, JHEP 10 (2005) 085 [hep-th/0508133] [INSPIRE].

[6] G. Aldazabal, P.G. Camara, A. Font and L. Ibáñez, More dual fluxes and moduli fixing, JHEP 05 (2006) 070 [hep-th/0602089] [INSPIRE].

[7] G. Aldazabal, P.G. Camara and J. Rosabal, Flux algebra, Bianchi identities and Freed-Witten anomalies in F-theory compactifications, Nucl. Phys. B 814 (2009) 21 [arXiv:0811.2900] [INSPIRE].

[8] G. Aldazabal, E. Andres, P.G. Camara and M. Graña, U-dual fluxes and generalized geometry, JHEP 11 (2010) 083 [arXiv: 1007.5509] [INSPIRE].

[9] N. Hitchin, Generalized Calabi-Yau manifolds, Quart. J. Math. Oxford Ser. 54 (2003) 281 [math/0209099] [INSPIRE].

[10] M. Gualtieri, Generalized complex geometry, math/0401221 [INSPIRE].

[11] M. Graña, R. Minasian, M. Petrini and D. Waldram, T-duality, generalized geometry and non-geometric backgrounds, JHEP 04 (2009) 075 [arXiv:0807.4527] [INSPIRE].

[12] S. Hellerman, J. McGreevy and B. Williams, Geometric constructions of nongeometric string theories, JHEP 01 (2004) 024 [hep-th/0208174] [INSPIRE].

[13] A. Dabholkar and C. Hull, Duality twists, orbifolds and fluxes, JHEP 09 (2003) 054 [hep-th/0210209] [INSPIRE]. 
[14] C. Hull, A geometry for non-geometric string backgrounds, JHEP 10 (2005) 065 [hep-th/0406102] [INSPIRE].

[15] C. Hull and R. Reid-Edwards, Flux compactifications of string theory on twisted tori, Fortsch. Phys. 57 (2009) 862 [hep-th/0503114] [INSPIRE].

[16] A. Dabholkar and C. Hull, Generalised T-duality and non-geometric backgrounds, JHEP 05 (2006) 009 [hep-th/0512005] [INSPIRE].

[17] C.M. Hull, Doubled geometry and t-folds, JHEP 07 (2007) 080 [hep-th/0605149] [INSPIRE].

[18] D.S. Berman, N.B. Copland and D.C. Thompson, Background field equations for the duality symmetric string, Nucl. Phys. B 791 (2008) 175 [arXiv:0708.2267] [InSPIRE].

[19] C. Hull and R. Reid-Edwards, Gauge symmetry, T-duality and doubled geometry, JHEP 08 (2008) 043 [arXiv:0711.4818] [INSPIRE].

[20] C. Hull and R. Reid-Edwards, Non-geometric backgrounds, doubled geometry and generalised T-duality, JHEP 09 (2009) 014 [arXiv:0902.4032] [INSPIRE].

[21] D.S. Berman and M.J. Perry, Generalized geometry and M-theory, JHEP 06 (2011) 074 [arXiv: 1008.1763] [INSPIRE].

[22] D.S. Berman, H. Godazgar and M.J. Perry, SO $(5,5)$ duality in M-theory and generalized geometry, Phys. Lett. B 700 (2011) 65 [arXiv:1103.5733] [InSPIRE].

[23] S. Kachru, M.B. Schulz, P.K. Tripathy and S.P. Trivedi, New supersymmetric string compactifications, JHEP 03 (2003) 061 [hep-th/0211182] [INSPIRE].

[24] D. Andriot, M. Larfors, D. Lüst and P. Patalong, A ten-dimensional action for non-geometric fluxes, JHEP 09 (2011) 134 [arXiv:1106.4015] [INSPIRE].

[25] C. Hull and B. Zwiebach, Double field theory, JHEP 09 (2009) 099 [arXiv:0904.4664] [INSPIRE].

[26] C. Hull and B. Zwiebach, The gauge algebra of double field theory and courant brackets, JHEP 09 (2009) 090 [arXiv:0908.1792] [INSPIRE].

[27] O. Hohm, C. Hull and B. Zwiebach, Background independent action for double field theory, JHEP 07 (2010) 016 [arXiv: 1003.5027] [INSPIRE].

[28] O. Hohm, C. Hull and B. Zwiebach, Generalized metric formulation of double field theory, JHEP 08 (2010) 008 [arXiv: 1006.4823] [INSPIRE].

[29] O. Hohm and S.K. Kwak, Massive type II in double field theory, arXiv:1108.4937 [INSPIRE].

[30] O. Hohm and S.K. Kwak, Double field theory formulation of heterotic strings, JHEP 06 (2011) 096 [arXiv:1103.2136] [INSPIRE].

[31] O. Hohm, S.K. Kwak and B. Zwiebach, Double field theory of type II strings, JHEP 09 (2011) 013 [arXiv:1107.0008] [INSPIRE].

[32] O. Hohm, S.K. Kwak and B. Zwiebach, Unification of type II strings and T-duality, arXiv:1106.5452 [INSPIRE].

[33] C. Albertsson, S.-H. Dai, P.-W. Kao and F.-L. Lin, Double field theory for double D-branes, JHEP 09 (2011) 025 [arXiv: 1107.0876] [INSPIRE].

[34] I. Jeon, K. Lee and J.-H. Park, Stringy differential geometry, beyond Riemann, Phys. Rev. D 84 (2011) 044022 [arXiv:1105.6294] [INSPIRE].

[35] I. Jeon, K. Lee and J.-H. Park, Double field formulation of Yang-Mills theory, Phys. Lett. B 701 (2011) 260 [arXiv:1102.0419] [INSPIRE]. 
[36] I. Jeon, K. Lee and J.-H. Park, Differential geometry with a projection: application to double field theory, JHEP 04 (2011) 014 [arXiv: 1011.1324] [INSPIRE].

[37] D.C. Thompson, Duality invariance: from M-theory to double field theory, JHEP 08 (2011) 125 [arXiv:1106.4036] [INSPIRE].

[38] O. Hohm and S.K. Kwak, Frame-like geometry of double field theory, J. Phys. A A 44 (2011) 085404 [arXiv:1011.4101] [InSPIRE].

[39] S.K. Kwak, Invariances and equations of motion in double field theory, JHEP 10 (2010) 047 [arXiv: 1008.2746] [INSPIRE].

[40] J. Scherk and J.H. Schwarz, How to get masses from extra dimensions, Nucl. Phys. B 153 (1979) 61 [INSPIRE].

[41] N. Kaloper and R.C. Myers, The odd story of massive supergravity, JHEP 05 (1999) 010 [hep-th/9901045] [INSPIRE].

[42] J. Schon and M. Weidner, Gauged N=4 supergravities, JHEP 05 (2006) 034 [hep-th/0602024] [INSPIRE].

[43] D. Andriot, Heterotic string from a higher dimensional perspective, arXiv:1102.1434 [INSPIRE].

[44] G. Aldazabal, D. Marques, C. Núñez and J.A. Rosabal, On type IIB moduli stabilization and $N=4,8$ supergravities, Nucl. Phys. B 849 (2011) 80 [arXiv:1101.5954] [INSPIRE].

[45] G. Dibitetto, A. Guarino and D. Roest, How to halve maximal supergravity, JHEP 06 (2011) 030 [arXiv:1104.3587] [INSPIRE].

[46] J.-P. Derendinger, P. Petropoulos and N. Prezas, Axionic symmetry gaugings in $N=4$ supergravities and their higher-dimensional origin, Nucl. Phys. B $\mathbf{7 8 5}$ (2007) 115 [arXiv:0705.0008] [INSPIRE].

[47] W. Siegel, Superspace duality in low-energy superstrings, Phys. Rev. D 48 (1993) 2826 [hep-th/9305073] [INSPIRE].

[48] W. Siegel, Two vierbein formalism for string inspired axionic gravity, Phys. Rev. D 47 (1993) 5453 [hep-th/9302036] [INSPIRE].

[49] A. Coimbra, C. Strickland-Constable and D. Waldram, Supergravity as generalised geometry I: type II theories, arXiv:1107.1733 [INSPIRE].

[50] G. Dall'Agata, G. Villadoro and F. Zwirner, Type-IIA flux compactifications and $N=4$ gauged supergravities, JHEP 08 (2009) 018 [arXiv:0906.0370] [INSPIRE].

[51] T. Danckaert, J. Louis, D. Martinez-Pedrera, B. Spanjaard and H. Triendl, The $N=4$ effective action of type IIA supergravity compactified on SU(2)-structure manifolds, JHEP 08 (2011) 024 [arXiv:1104.5174] [INSPIRE].

[52] T. Buscher, A symmetry of the string background field equations, Phys. Lett. B 194 (1987) 59 [InSPIRE].

[53] A. Giveon, M. Porrati and E. Rabinovici, Target space duality in string theory, Phys. Rept. 244 (1994) 77 [hep-th/9401139] [INSPIRE].

[54] G. Dall'Agata, N. Prezas, H. Samtleben and M. Trigiante, Gauged supergravities from twisted doubled tori and non-geometric string backgrounds, Nucl. Phys. B 799 (2008) 80 [arXiv: 0712.1026] [INSPIRE].

[55] D. Geissbuhler, Double field theory and $N=4$ gauged supergravity, arXiv:1109.4280 [INSPIRE]. 